\title{
Perturbation of Macaque Supplementary Motor Area Produces Context-Independent Changes in the Probability of Movement Initiation
}

\author{
Andrew J. Zimnik, ${ }^{1,2 *}$ Antonio H. Lara, ${ }^{1 *}$ and Mark M. Churchland ${ }^{1,2,3,4}$ \\ ${ }^{1}$ Department of Neuroscience, ${ }^{2}$ Zuckerman Institute, Columbia University, New York 10027, New York, ${ }^{3}$ Kavli Institute for Brain Science, Columbia \\ University Medical Center, New York 10032, New York, and ${ }^{4}$ Grossman Center for the Statistics of Mind, Columbia University, New York 10027, \\ New York
}

The contribution of the supplementary motor area (SMA) to movement initiation remains unclear. SMA exhibits premovement activity across a variety of contexts, including externally cued and self-initiated movements. Yet SMA lesions impair initiation primarily for self-initiated movements. Does SMA influence initiation across contexts or does it play a more specialized role, perhaps contributing only when initiation is less dependent on external cues? To address this question, we perturbed SMA activity via microstimulation at variable times before movement onset. Experiments used two adult male rhesus monkeys trained on a reaching task. We used three contexts that differed regarding how tightly movement initiation was linked to external cues. Movement kinematics were not altered by microstimulation. Instead, microstimulation induced a variety of changes in the timing of movement initiation, with different effects dominating for different contexts. Despite their diversity, these changes could be explained by a simple model where microstimulation has a stereotyped impact on the probability of initiation. Surprisingly, a unified model accounted for effects across all three contexts, regardless of whether initiation was determined more by external cues versus internal considerations. All effects were present for stimulation both contralateral and ipsilateral to the moving arm. Thus, the probability of initiating a pending movement is altered by perturbation of SMA activity. However, changes in initiation probability are independent of the balance of internal and external factors that establish the baseline initiation probability.

Key words: macaque; microstimulation; reaching; supplementary motor area

Significance Statement

The role of the supplementary motor area (SMA) in initiating movement remains unclear. Lesion experiments suggest that SMA makes a critical contribution only for self-initiated movements. Yet SMA is active before movements made under a range of contexts, suggesting a less-specialized role in movement initiation. Here, we use microstimulation to probe the role of SMA across a range of behavioral contexts that vary in the degree to which movement onset is influenced by external cues. We demonstrate that microstimulation produces a temporally stereotyped change in the probability of initiation that is independent of context. These results argue that SMA participates in the computations that lead to movement initiation and does so across a variety of contexts.

\section{Introduction}

The role of the supplementary motor area (SMA) in movement initiation remains debated. SMA lesions impair self-initiated

Received Sept. 11, 2018; revised Jan. 16, 2019; accepted Feb. 4, 2019.

Author contributions: A.H.L. and M.M.C. designed the experiments; A.J.Z. and A.H.L. performed the experiments; A.J.Z. analyzed the data; A.J.Z. wrote the manuscript; A.J.Z., A.H.L., and M.M.C. edited the manuscript.

This work was supported by National Institute of Neurological Disorders and Stroke 1DP2NS083037, National Institutes of Health CRCNS R01NS100066, National Institute of Neurological Disorders and Stroke 1U19NS104649, the Sloan Foundation, the Simons Foundation (SCGB\#325233 and SCGB\#542957), the Grossman Center for the Statistics of Mind, the McKnight Foundation P30 EY019007, a Klingenstein-Simons Fellowship, the Searle Scholars Program, and National Science Foundation Graduate Research Fellowship DGE 1644869. We thank Yanina Pavlova and Sean Perkins for technical support. movements in humans (Krainik et al., 2001) and monkeys (Thaler et al., 1995) but not movements prompted by external cues (Chen et al., 1995). These observations suggest that SMA plays a specialized role, contributing primarily when initiation is based on internal considerations. Yet physiological recordings do not obviously support such specialization. Functional imaging in humans has produced conflicting reports that SMA is more active

The authors declare no competing financial interests.

${ }^{*}$ A.J.Z. and A.H.L. contributed equally to this work.

Correspondence should be addressed to Mark M. Churchland at mc3502@columbia.edu.

https://doi.org/10.1523/JNEUROSCI.2335-18.2019

Copyright $\odot 2019$ the authors 
(Jenkins et al., 2000) or similarly active (Cunnington et al., 2002; Sakata et al., 2017) during self-initiated versus externally cued contexts. In monkeys performing externally cued reaches, SMA is no less metabolically active than motor cortex (Picard and Strick, 2003). Similarly, single-neuron recordings reveal that SMA is active during both self-initiated and externally cued contexts (Romo and Schultz, 1987; Kurata and Wise, 1988; Thaler et al., 1988). Yet while the SMA population as a whole is active across contexts, single SMA-neuron responses show robust contextual dependence (Lara et al., 2018b). Prior results therefore suggest three possibilities. First, SMA may influence movement initiation primarily when initiation is determined by internal considerations. Second, SMA may influence movement initiation in different ways for different contexts (e.g., promoting movement in some contexts and inhibiting it in others). Third, although computations in SMA may be context-dependent, they may share a final influence on initiation that is present and consistent across contexts.

Here, we probe the role of SMA in movement initiation by using microstimulation to perturb activity before movement onset. Prior work has established that perturbations of SMA activity can impact initiation. Intrasurgical stimulation can cause an "urge to move" (Fried et al., 1991), and SMA cooling increases reaction times (RTs) (Tanji et al., 1985). Applying transcranial direct current stimulation over SMA for a period of minutes produces lasting poststimulation changes in both RT and the ability to withhold a reach (Hayduk-Costa et al., 2013; Carlsen et al., 2015). Additionally, intracranial stimulation of SMA can increase RTs in patients (Moore et al., 2018). We wished to determine the impact of spatially and temporally localized SMA microstimulation and to ask whether that impact varies depending on how tightly initiation is tied to external stimuli.

We examined the impact of SMA stimulation in monkeys trained to perform center-out reaches under three contexts. In the self-initiated context, monkeys were free to reach upon target onset but received larger rewards if they chose to wait. In the cueinitiated context, an explicit go cue prompted movement initiation. In the quasi-automatic context, moving targets evoked intercepting movements with short, narrowly distributed RTs. Thus, the three contexts evoked initiation that was loosely, tightly, or very tightly linked to sensory cues. We have previously shown that SMA activity, but not motor cortex activity or muscle activity, covaries robustly across these three contexts (Lara et al., 2018b).

We found that microstimulation, delivered shortly before the typical time of movement initiation, increased RTs across all three contexts. Notably, this was true even for the quasiautomatic context, where initiation was reactive, low-latency, and tightly linked to sensory cues. Yet the increase in RT was smaller for the quasi-automatic context than for the other two contexts. Furthermore, we observed an opposing effect that was sizeable only for the self-initiated context: RTs became shorter when microstimulation was delivered well in advance of typical initiation times. Thus, analysis of RT initially appeared to support the hypothesis that SMA stimulation has a context-dependent effect on initiation. However, the RT produces ambiguities when used as a measure of the tendency to initiate movement. A simple change in the probability of initiation can yield different RT effects, depending on the current and preceding baseline probabilities of initiation. We therefore focused directly on the probability of initiation at each moment in time following stimulation.

Surprisingly, effects of microstimulation were well explained by an entirely context-independent impact of microstimulation on the probability of initiation. Specifically, we considered that microstimulation might multiplicatively alter the odds of initia- tion (e.g., doubling or halving those odds). Assessed from this perspective, effects of stimulation were remarkably contextindependent. In all contexts, stimulation produced an immediate reduction in the odds of initiation, followed by an elevation. These context-independent changes in initiation probability naturally produced the context-dependent changes in RT.

In summary, perturbation of SMA activity impacted the probability that a pending movement would be initiated. That impact was surprisingly stereotyped and simple: it depended only on the time after stimulation and was essentially independent of contextual factors. In particular, the same model accounted for the impact of stimulation regardless of whether initiation was determined by internal considerations related to reward (the selfinitiated context), by a standard go cue (the cue-initiated context), or by a salient external cue that evoked short-latency, reactive movements (the quasi-automatic context). These results argue that SMA contributes to movement initiation across a range of contexts.

\section{Materials and Methods}

Subjects and task. Subjects were 2 adult, male macaque monkeys (Macaca mulatta), weighing $10-13 \mathrm{~kg}$. Daily fluid intake was regulated to maintain motivation to perform the task for juice reward. Animal protocols were in accord with the National Institutes of Health guidelines and approved by Columbia University Institutional Animal Care and Use Committee (Protocol AC-AAAQ7409).

Subjects sat in a primate chair facing an LCD display $(60 \mathrm{~Hz}$ refresh rate) and performed a unimanual, center-out reaching task with the right arm while the left arm and head were comfortably restrained. Hand position was tracked optically via a reflective bead placed between the third and fourth digits (Polaris, Northern Digital). The timing of displayed stimuli was monitored via photodetector (Thorlabs). The subject initiated each trial by touching and holding a central touch-point. Touch-point color indicated the context of the upcoming trial (blue represents self-initiated; red represents cue-initiated; yellow represents quasi-automatic). After the touch-point was held for 450-550 ms (randomized), a 10-mm-diameter circular target appeared in one of eight possible locations arranged radially around the touch-point. Target color matched touch-point color. Targets appeared $130 \mathrm{~mm}$ from the touch-point for the self-initiated and cue-initiated contexts. For the quasi-automatic context, targets appeared $40 \mathrm{~mm}$ from the touchpoint (as described below, subsequent target motion increased this distance to $\sim 130 \mathrm{~mm}$ ). Target directions and contexts were interleaved and presented in random order within blocks. The subsequent block began after all combinations of target direction and context were successfully completed.

In the self-initiated context, the target slowly and linearly grew in size, starting upon its appearance and ending when the reach began. Maximum target size of $30 \mathrm{~mm}$ was achieved $1200 \mathrm{~ms}$ after target appearance. The reward for a successful reach grew exponentially, beginning with 1 drop and attaining a maximum of 8 drops after $1200 \mathrm{~ms}$. Monkeys were free to reach as soon as the target was displayed and were required to begin their reach within $1500 \mathrm{~ms}$. Reaches were required to be $<500 \mathrm{~ms}$ in duration and to land within an 18-mm-radius window centered on the target. Reward was delivered if the target was held, with minimal further hand motion, for $600 \mathrm{~ms}$.

In the cue-initiated context, after a randomized delay period $(0-1000$ $\mathrm{ms}$ ), target size suddenly increased to $30 \mathrm{~mm}$ and the central touch-point simultaneously disappeared. These events served as a go cue, and reaches were required to be initiated within $500 \mathrm{~ms}$. Requirements for movement duration, accuracy, and the target hold period were the same as for the self-initiated context.

In the quasi-automatic context, after a randomized delay period ( 0 $1000 \mathrm{~ms}$ ), the target suddenly began moving radially away from the central touch-point at $25 \mathrm{~cm} / \mathrm{s}$. Target motion ceased if a reach landed on the target mid-flight $(16 \mathrm{~mm} \times 20 \mathrm{~mm}$ radius acceptance ellipse; long axis aligned with target motion). If successfully captured, the target grew to 30 
$\mathrm{mm}$ and reward was delivered after a $600 \mathrm{~ms}$ hold period (as above). If the target was not intercepted (reach duration or RT was too long, or the reach missed), the target continued moving until off the screen (which occurred $630 \mathrm{~ms}$ after target-motion onset) and no reward was delivered. Target starting position and speed were carefully selected during training such that target-capture occurred $\sim 130 \mathrm{~mm}$ from the touch-point, matching reach extent in the self-initiated and cue-initiated contexts.

Microstimulation. Cylindrical recording chambers (19 $\mathrm{mm}$ inner diameter, Crist Instruments) were implanted above the skull to allow access to SMA. Chamber positioning was guided by structural magnetic resonance images taken before implantation. For each monkey, chambers were moved mid-experiment to allow stimulation of the other hemisphere. Electrodes were introduced via small holes drilled by hand in the chamber, which then healed following experiments. Before stimulation, neural recordings were performed to verify the presence of robust delay period activity (Lara et al., 2018b). We observed such activity in both hemispheres, confirming that SMA is active for both ipsilateral and contralateral movements (Kermadi et al., 1998; Nakayama et al., 2015).

Microstimulation ( $333 \mathrm{~Hz}, 200 \mathrm{~ms}$, biphasic pulses) was generated by a programmable pulse generator and electrical stimulus generator (neuro/Craft StimPulse Stimulator, FHC). Stimulation was delivered via tungsten microelectrodes $(\sim 1-2 \mathrm{M} \Omega$; FHC), lowered into position by a motorized microdrive (Narishige). For each stimulation site, we estimated the threshold current for evoking movement based on visual observation and/or manual palpation. We observed a broad range of stimulation thresholds, from 10 to $>150 \mu$ A. Stimulation was often performed at multiple sites along each penetration; the above range will thus include both deeper and superficial layers. The lower end of the observed range is consistent with previous reports (Macpherson et al., 1982; Mitz and Wise, 1987). Thresholds in the upper half of this range have not been explicitly reported but can be inferred from prior work. For example, in Mitz and Wise (1987), $\sim 40 \%$ of sites had thresholds $>65 \mu \mathrm{A}$ and were not tested further. Similarly, in Macpherson et al. (1982), $60 \%$ of sites had thresholds $>30 \mu \mathrm{A}$ and were not tested further. The broad range of thresholds we observed in SMA is also consistent with the broad range of thresholds previously observed in dorsal premotor cortex (Churchland and Shenoy, 2007).

Microstimulation elicited movements of the distal and proximal arm, including muscles of the shoulder girdle (e.g., deltoid and pectoralis). When examining the impact of microstimulation on movement initiation, we used currents set to $80 \%-90 \%$ of threshold. In a few cases, we examined effects at sites where we were not able to directly elicit movement but which were near microexcitable sites. In these cases, current was set near the upper range of thresholds for those nearby sites.

A subset of trials (25\%) were randomly chosen to be stimulation trials (9854 trials and 4591 trials, Monkeys B and A). Microstimulation onset was determined by a draw from a uniform distribution $(0-1000 \mathrm{~ms})$ starting with target onset. This broad distribution allowed us to probe the effect of microstimulation at different times relative to when movement initiation typically occurred and ensured that stimulation onset was not predictive of the go cue. If the monkey began moving before the chosen time of stimulation onset, stimulation was not delivered. Thus, on trials where stimulation was delivered, it always began between target and movement onset.

We were interested in the impact of microstimulation on the initiation of a pending movement that was already instructed and known. For the self-initiated context, this was naturally true given the monkeys' behavior: initiation essentially always occurred $>500 \mathrm{~ms}$ after target onset. Thus, the target was known well before initiation became likely. For the cue-initiated and quasi-automatic contexts, this was not always true: the delay period could be as short as $0 \mathrm{~ms}$, and movement onset could occur with low latency. We therefore analyzed only trials with delay periods $>200 \mathrm{~ms}$. Shorter delays were still used to encourage early and consistent preparation.

Detecting movement onset. For purposes of behavioral control and reward, movement onset was detected online as the moment when hand speed exceeded $20 \mathrm{~mm} / \mathrm{s}$ ( $\sim 2 \%$ of peak speed). All analyses used a more accurate, offline estimate of movement onset. We found that a pure threshold did not strike an optimal balance between estimating the very first moment the hand began to move and being robust to slight trial-totrial differences in the early temporal profile of hand velocity. We thus used the following procedure (Lara et al., 2018b). For each trial, we estimated the moment of peak acceleration: $t_{\text {peakAcc }}$. This identified an early feature of the response that occurred in a consistent fashion across trials; aligning trials on $t_{\text {peakAcc }}$ caused velocity profiles to be well aligned. For each condition, we aligned all trials in that fashion and computed the average speed. From the average speed profile, we found the time interval, $t_{\text {corr }}$, between when speed crossed a low threshold ( $1 \%$ of its peak) and the time of alignment, $t_{\text {peakAcc }}$. The RT for each trial was then $t_{\text {peakAcc }}-$ $t_{\text {corr }}$. This method estimated well the moment when hand velocity first began to change while also ensuring that kinematic events (e.g., the bellshaped hand-velocity profile) were well aligned when trials were aligned on movement onset. The location of the reach endpoint was calculated at the time when hand speed first fell to $<20 \mathrm{~mm} / \mathrm{s}$. Initial reach direction was calculated when the hand reached peak speed.

Estimating $\mathrm{P}_{0}(\mathrm{t})$ and $\mathrm{P}_{\text {stim }}\left(\mathrm{t}, \mathrm{t}_{\mathrm{s}}\right)$. We define $\mathrm{P}_{0}(t)$ as the probability of initiating a reach at time $t$ given that a reach has not been initiated before $t$. That is, $P_{0}(t)$ is the hazard rate of reach initiation. Because the probability of initiating a reach at any particular time $t$ is infinitesimal, we compute the probability of initiation within a $150 \mathrm{~ms}$ window as follows:

$$
P_{0}(t)=P\left(T_{m} \in[t, t+150] \mid T_{m} \geq t\right)
$$

where $T_{m}$ is the recorded time of movement onset. We estimated $P_{0}(t)$ by taking all trials where movement was initiated after time $t$ and computing the proportion initiated within the interval $[t, t+150]$. To obtain a reliable estimate, $P_{0}(t)$ was estimated only for values of $t$ where there were at least 80 trials where initiation had not yet occurred by time $t$. The 150 ms interval was also helpful for accurately estimating $P_{0}(t)$, but intervals as short as $50 \mathrm{~ms}$ yielded very similar overall results.

We define the probability of initiation following stimulation as follows:

$$
P_{\text {stim }}\left(t, t_{s}\right)=P\left(T_{m} \in[t, t+150] \mid T_{m} \geq t, T_{s} \in\left[t_{s}, t_{s}+150\right]\right)
$$

Where $T_{s}$ is the time of stimulation. $P_{0}(t)$ and $P_{\text {stim }}\left(t, t_{s}\right)$ were calculated every $10 \mathrm{~ms}$. $P_{\text {stim }}\left(t, t_{s}\right)$ was estimated only for values of $t$ and $t_{s}$ that yielded at least 20 observations. We define the change in the probability of moving as follows:

$$
\Delta P_{\text {stim }}\left(t, t_{s}\right)=P_{\text {stim }}\left(t, t_{s}\right)-P_{0}(t)
$$

Estimating the stimulation kernel. We used a multiplicative model that attempts to predict $\Delta P_{\text {stim }}\left(t, t_{s}\right)$ given $P_{0}(t)$ and a kernel, $k\left(t-t_{s}\right)$, capturing the impact of stimulation at time $t_{s}$ on the odds of initiating at time $t$. The odds of initiation is the ratio of the probability of initiating to the probability of not initiating as follows:

$$
O_{0}(t)=\frac{P_{0}(t)}{1-P_{0}(t)}
$$

The impact of stimulation is modeled as follows:

$$
\hat{O}\left(t, t_{s}\right)=O_{0}(t) k\left(t-t_{s}\right),
$$

where $\hat{O}\left(t, t_{s}\right)$ is the predicted odds of initiation at time $t$ given stimulation at time $t_{s}$. Values of $k>1$ increase the odds of initiation, whereas values of $k<1$ decrease the odds of initiation. The predicted probability of initiation is then:

$$
\widehat{\Delta P}_{\text {stim }}\left(t, t_{s}\right)=\frac{\hat{O}\left(t, t_{s}\right)}{1+\hat{O}\left(t, t_{s}\right)} .
$$

We fit $k$ to minimize the objective function as follows:

$$
\sum_{t, t_{s}}^{N, M}\left(\widehat{P P}_{\text {stim }}\left(t, t_{s}\right)-\Delta P_{\text {stim }}\left(t, t_{s}\right)\right)^{2}+\alpha \max _{t}\left(\frac{d^{2} k}{d t^{2}}\right)^{2} .
$$

The first term in the objective function encourages accuracy, whereas the second term regularizes the solution by encouraging a kernel that 
changes smoothly with time. We enforced $k\left(t-t_{s}\right)=1$ for $t \leq t_{s}$, reflecting the assumption that stimulation cannot have acausal effects. We enforced $k\left(t-t_{s}\right)=1$ for $t-t_{s}>$ 1000 , reflecting both the assumption that stimulation likely has negligible impact after $1 \mathrm{~s}$ and the practical reality that we lack sufficient data to meaningfully constrain a very long kernel. In practice, fitting typically returned values of $k(0)$ and $k(1000)$ near unity, even if not enforced. The constant $\alpha$ was set to $N M / 100$, where $N$ and $M$ are the number of values of $t$ and $t_{s}$ for which we measured $P_{\text {stim }}\left(t, t_{s}\right)$. In practice, the value of $\alpha$ had a very modest impact on the shape of the resulting kernel.

Statistical tests. Standard statistical tests, most often paired $t$ tests, are used when appropriate, as indicated in Results. The relevant degrees of freedom (number of trials, stimulation sites, conditions) differs by comparison and is given either as the test is described or when documenting the numbers of stimulated sites and stimulated trials. Bootstrap (resampling) methods were used in a number of instances to estimate the statistical reliability of estimated quantities (see Evaluating fit quality).

Evaluating fit quality. Fit quality was quantified in terms of $R^{2}$ : the proportion of variance in $\Delta P_{\text {stim }}\left(t, t_{s}\right)$ accounted for by the model prediction. We checked for overfitting by redrawing data (across sites) 1000 times and verifying that the resulting kernel remained similar. This approach was used for analyses inquiring about the statistical reliability of results across stimulation sites and allowed plotting of SEs on our estimate of the kernel.

We also used a bootstrap procedure to ask what the range of kernels would look like, due to sampling error across trials, if there were no effect of stimulation. For each of 100 repetitions, we replaced the initiation time on each stimulated trial with the initiation time on a randomly drawn unstimulated trial, selected to have sufficient time after target (delay + RT) that stimulation at the relevant time could have been delivered. We then analyzed the data as usual and fit the model. The mean and SD of the resulting kernels indicates the range of kernels expected by chance.

The above approaches address the issue of overfitting. The converse concern is potentially larger: the measured $R^{2}$ may underestimate fit quality given sampling error in the estimate of $\Delta P_{\text {stim }}\left(t, t_{s}\right)$. To estimate a rough upper limit on how well a good model should fit, we asked how well redrawn data would "fit" the original data. We generated surrogate datasets by redrawing with replacement from all recorded trials, preserving the number of stimulated and nonstimulated trials. We then calculated the $R^{2}$ between the observed data and each of 1000 surrogate datasets and calculated the mean and 95\% CIs for the resulting distribution. For infinite trials, this approximate upper limit would be unity. For the empirical data, the upper limit ranged from $\sim 0.8$ to 0.95 depending on the monkey and context.

\section{Results}

\section{Task and behavior}

Two monkeys (Monkeys Ba and Ax) executed radial reaches in eight directions across three contexts. Contexts were cued by target color and differed regarding the cues and task constraints governing when movement should be initiated. SMA has been proposed to contribute preferentially when initiation is prompted more by internal considerations versus external cues (Jenkins et al., 2000; Passingham et al., 2010). We note that goal-directed voluntary movements are essentially never purely self-initiated; initiation will almost always relate to some preceding instruction or information. However, movements vary greatly in the degree to which initiation timing is linked to external sensory cues. We assessed the tightness of that link via the RT: the latency between the cue indicating that movement is allowed and subsequent reach initiation. Shorter/more-tightly distributed RTs were taken to indicate a tighter link between external cues and movement initiation.

In the self-initiated context (Fig. 1A, blue), monkeys were free to reach upon target appearance, but waiting longer yielded increased reward up to a maximum at $1200 \mathrm{~ms}$. Growing reward was mirrored by increasing target diameter, eliminating any need to estimate elapsed time. RTs were long with broad SDs: $1069 \pm$ $142 \mathrm{~ms}$ (mean $\pm \mathrm{SD}$ ) for Monkey Ba and $1023 \pm 106 \mathrm{~ms}$ for Monkey Ax (Fig. $1 B, C$, blue line). The considerable RT variability presumably reflects competition between internal factors (desire for immediate reward versus desire for large reward) with different factors dominating on different trials. Consistent with this interpretation, the distribution of self-initiated RTs during training was very sensitive to the reward schedule. The selfinitiated context does not provide a situation as unrestricted as in 
Thaler et al. (1995), where monkeys could go minutes between self-initiated movements. Instead, the self-initiated context gives monkeys considerable control over when to initiate while also providing a well-defined temporal window upon which analysis could concentrate.

The cue-initiated context (Fig. 1A, red) emulated the standard instructed-delay paradigm: a variable delay period $(0-1000 \mathrm{~ms})$ separated target onset from a go cue, indicated by an instantaneous increase in target size and the simultaneous disappearance of the central touch point. After the go cue, monkeys still had modest leeway regarding the exact moment of initiation; RTs up to $500 \mathrm{~ms}$ were allowed. Yet monkeys generally reached promptly out of desire for immediate reward: RTs were $238 \pm 49 \mathrm{~ms}$ and $222 \pm 37$ ms (mean \pm SD for Monkeys Ba and Ax; Fig. $1 B, C$, red line). Thus, relative to the self-initiated context, initiation was more tightly locked to an external cue. Initiation occurred closer in time to the key cue (RTs were approximately one-fourth as long) and was less temporally variable (SDs were approximately one-third as wide).

The quasi-automatic context (Fig. $1 C$, yellow) was similar to the cue-initiated context and also included a $0-1000$ ms delay. However, the go cue was the onset of target motion along a radial path toward the screen's edge. Success required executing a reach that intercepted the target mid-flight. Moving targets are known to naturally produce reactive, short-RT, intercepting reaches (Perfiliev et al., 2010; Wong et al., 2017; Lara et al., 2018a). In keeping with that finding, RTs were $199 \pm 28 \mathrm{~ms}$ and $184 \pm 27 \mathrm{~ms}$ (mean $\pm \mathrm{SD}$ for Monkeys Ba and Ax; Fig. 1B,C, yellow line). Essentially no training was required to obtain short RTs; RTs dropped immediately upon the introduction of moving targets. Thus, the quasi-automatic context naturally evoked initiation that was tightly locked to a very salient external cue. Initiation occurred even closer in time to that cue than in the cue-initiated context, with even lower temporal variability. Given sizeable physiological delays $(\sim 130 \mathrm{~ms}$ including afferent, efferent, and EMG-to-velocity delays), initiation in the quasi-automatic context occurs with little or no time for internal deliberation. Thus, if SMA plays a specialized role in internally initiated movements, SMA would be expected to make little contribution to initiation in the quasi-automatic context.

Monkeys successfully completed the majority of trials for all three contexts: $94 \%$ and $99 \%$ of cue-initiated trials (Monkey Ba and Monkey Ax, respectively), $92 \%$ and $96 \%$ of self-initiated trials, and $86 \%$ and $87 \%$ of quasi-automatic trials. Reach trajectories were similar across contexts; the primary difference was a tendency for reach velocity to be slightly higher for quasiautomatic reaches (Fig. 2E,F). We have previously shown that these three contexts evoke similar patterns of muscle activity and similar patterns of neural activity in primary motor and premotor cortex (Lara et al., 2018b). In contrast, neural activity in SMA (recorded from sites interspersed with those stimulated in the present study) varies robustly across these three contexts (Lara et al., 2018b). The presence of differential neural activity raises the possibility that SMA could be making differential contributions to initiation across contexts. On the other hand, the fact that SMA is active for all contexts suggests that SMA might contribute to initiation in all contexts.

\section{Reach kinematics were largely unaffected by stimulation}

We delivered microstimulation (200 ms train of $333 \mathrm{~Hz}$ biphasic pulses) at 122 sites in SMA of 2 monkeys (Fig. 1D). Stimulation sites lay within a region where, during separate recordings, we observed robust preparatory and movement-related activity in this task. Task-related activity was observed with approximately equal magnitude both contralateral and ipsilateral to the reaching hand. The impact of stimulation was thus examined for both hemispheres (Monkey Ba: 66 contralateral and 11 ipsilateral sites; Monkey Ax: 24 contralateral and 21 ipsilateral sites). For each site, we first determined the threshold for directly eliciting movement. Thresholds varied broadly (Fig. $1 E$ ), from $10 \mu \mathrm{A}$ to above the highest tested current $(150 \mu \mathrm{A})$. During experiments, stimulation current was set below threshold. Unless otherwise stated, data are pooled across sites for statistical power. This yielded a total of 9854 and 4591 stimulated trials (Monkeys Ba and Ax), comprising $20 \%$ and $20 \%$ of all trials. Thresholds, and thus currents, tended to be higher for Monkey Ba. As will be described below, the impact of microstimulation was qualitatively similar for both monkeys, with some effects being larger in Monkey Ba and others being larger in Monkey Ax. Thus, there was not an obvious relationship between higher currents and larger effects.

We wished to assess the impact of stimulation on movement initiation, independent of any impact on the reaches themselves. Conveniently, stimulation had almost no impact on reach kinematics. Velocity profiles on stimulated trials were very similar to those on nonstimulated trials. The correlation between the average velocity trajectories for stimulated and nonstimulated trials was 0.998 and 0.995 (Monkeys Ba and Ax, mean across conditions). The primary effect of stimulation on kinematics was a small increase in reach-speed variability. Across monkeys/contexts, there was a $1.2 \%-9.9 \%$ increase in the SD of peak reach speed. This effect was significant ( $p<0.05$ for each monkey, paired $t$ test, calculated across 77 and 45 stimulation sites) for the cue-initiated and quasi-automatic contexts only. We also observed small and inconsistent changes in reach angle variability. For Monkey $\mathrm{Ba}$, microstimulation in the self-initiated context caused a small $(1 \%)$ but significant $(p<0.001$, paired $t$ test) tightening of the circular $\mathrm{SD}$ of reach angle, yet microstimulation in the other two contexts caused a small increase $(2 \%$ and $1 \%$, $p<0.001$ for both, paired $t$ test). For Monkey Ax, microstimulation had no impact on reach variability in the self-initiated context, caused a modest increase $(10 \%, p<0.001$, paired $t$ test $)$ in the cue-initiated context, and a small decrease $(3 \%, p<0.001$, paired $t$ test) in the quasi-automatic context. Thus, the impact of stimulation on reach kinematics was small and did not interfere with the ability to assess changes in the time of reach initiation.

\section{Microstimulation impacts RT}

We grouped data into early-stimulation trials (stimulation delivered well before initiation would typically occur) and latestimulation trials (stimulation delivered close to when initiation would typically occur). Because the distribution of initiation times differed across contexts, we defined early versus late relative to a common reference: the time when movement had begun on $10 \%$ of nonstimulated trials in that context. Early-stimulation trials were those where microstimulation began $700-550 \mathrm{~ms}$ before this reference, such that stimulation ended well before movement initiation became likely. Late-stimulation trials were those where microstimulation began $300-150 \mathrm{~ms}$ before the reference, such that stimulation occurred just when initiation was becoming likely.

For Monkey Ba, early and late stimulation trials constituted $12 \%$ and $17 \%$ of stimulated trials ( $3 \%$ and $4 \%$ of all trials). There were 1218 early stimulation trials: 353,442 , and 423 , for the self-initiated, cue-initiated, and quasi-automatic contexts. There were 1647 late stimulation trials: 426, 631, and 590, for the selfinitiated, cue-initiated, and quasi-automatic contexts. For Mon- 
key Ax, early and late stimulation trials constituted $13 \%$ and $16 \%$ of stimulated trials (3\% and $3 \%$ of all trials). There were 609 early stimulation trials: 161, 215, and 233, for the self-initiated, cue-initiated, and quasi-automatic contexts. There were 738 late stimulation trials: 170,318 , and 250 , for the self-initiated, cue-initiated, and quasiautomatic contexts.

Late stimulation delayed movement initiation, increasing RT relative to nonstimulated trials. This was true for both monkeys and all three contexts (Fig. $2 A-D ; p<0.001$ for all, two-sample $t$ test). Notably, SMA stimulation increased RT even for the quasi-automatic context, despite initiation being tightly linked to external cues. However, the RT increase was smaller for the quasi-automatic context than for the other two contexts, suggesting some potential interaction between context and the impact of stimulation.

Early stimulation had the opposite effect on RT, and effects depended even more strongly on context. For the selfinitiated context, early stimulation advanced movement initiation $(p<0.001$ and $p=0.002$ for Monkeys $\mathrm{Ba}$ and $\mathrm{Ax}$, two-sample $t$ test). This RT reduction was much smaller for the other two contexts: ranging from 1 to $9 \mathrm{~ms} ; p<0.05$ only for the quasi-automatic context.

We wished to rule out the possibility that changes in RT are secondary to changes in reach kinematics (e.g., changes in reach velocity might alter the estimate of movement onset). This is unlikely: we showed above that stimulation has little average impact on kinematics. However, it remains possible that early and late stimulations have sizeable but opposing effects that largely cancel out on average. This was not the case (Fig. 2E,F). Movement duration, initial reach direction, and endpoint variability were not significantly different between no-stimulation, earlystimulation, and late-stimulation trials (Fig. 2G). We did observe a small increase in endpoint error following early stimulation (data not shown): a $0.31 \mathrm{~mm}$ increase in mean error in Monkey $\mathrm{Ba}$ and a 0.85 $\mathrm{mm}$ increase in Monkey Ax (Fig. 2G; $p=$ 0.03 and $p<0.001$, respectively, twosample $t$ test). This increase in endpoint error did not impact task performance: the percentage of trials failed due to a missed target was unchanged (remaining at $1.4 \%$ for Monkey $\mathrm{Ba}$ and $0.3 \%$ for Monkey Ax). We also did not observe any interaction between reach direction and the impact of stimulation on RT. For example, the change in RT with stimulation
A

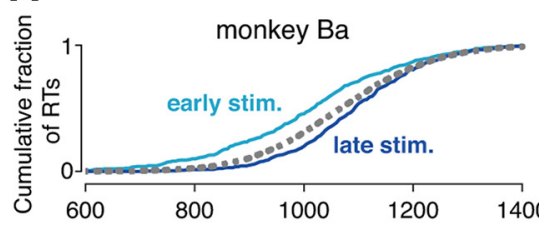

B
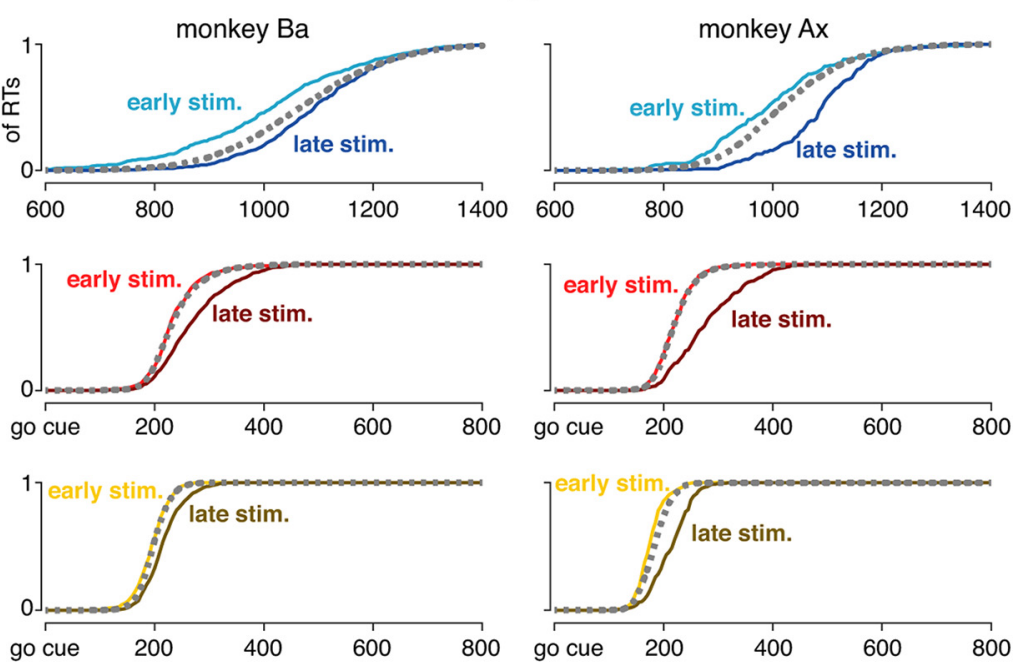

C
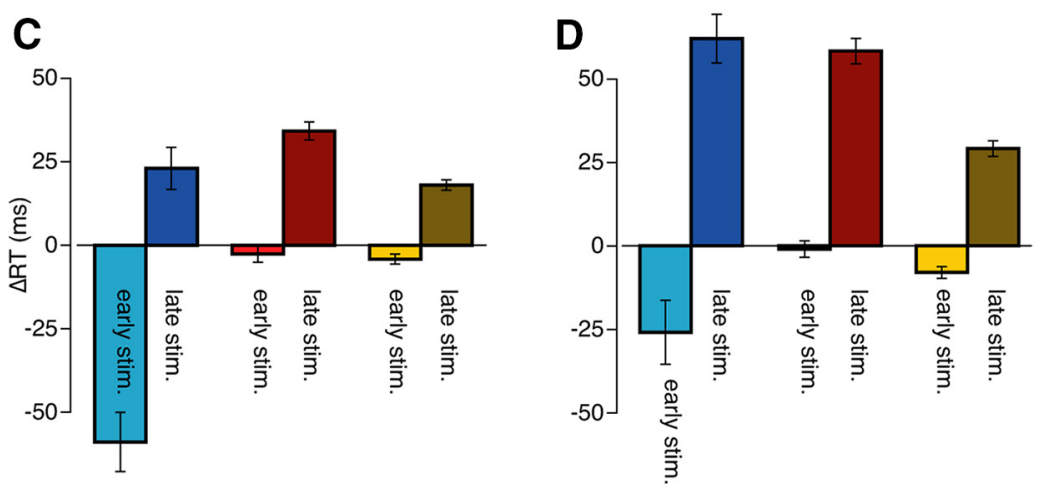

E

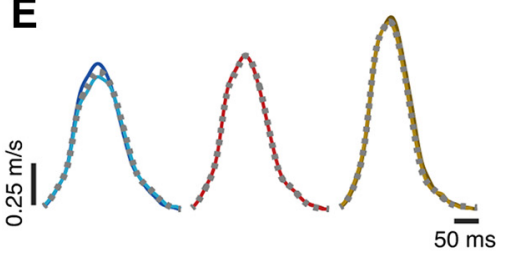

$\mathbf{F}$

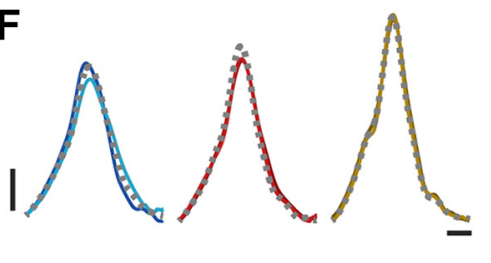

G
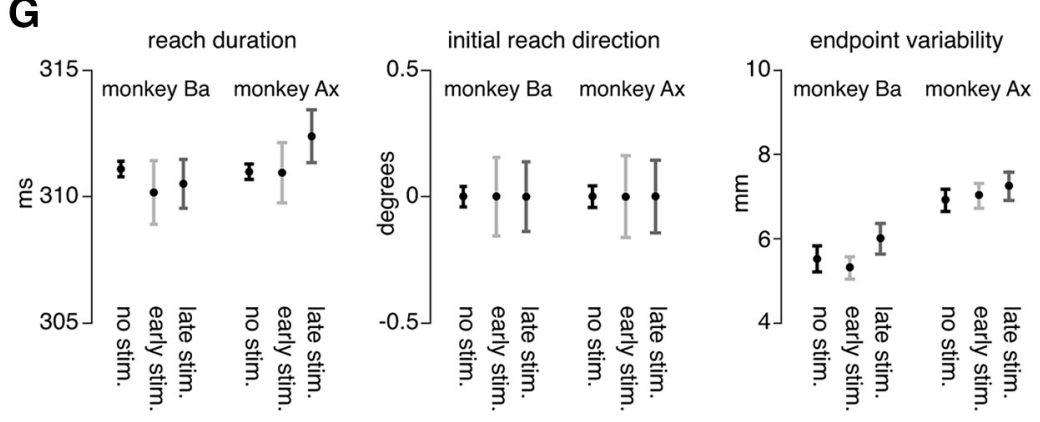

Figure 2. Microstimulation produces complex changes in RT, but not reach kinematics. $A, B$, Cumulative RT distributions for trials with no stimulation (dashed gray traces), early stimulation (light colored traces), and late stimulation (dark colored traces). $C, D$, Summary of the above effects in terms of changes in mean RT. Error bars indicate SEs. The mean \pm SE were computed across trials. For all comparisons, the RT of nonstimulated trials was computed using only trials where the delay period length and RT would have allowed stimulation to be delivered. This eliminates potential biases; for example, late stimulation might otherwise appear to increase RT simply because late stimulation implies that movement has not yet been initiated. $E, F$, Mean speed profiles for reaches to one representative target (target located rightward of central position) for the three contexts. Dashed gray represents no stimulation. Light colored traces represent early stimulation. Dark colored traces represent late stimulation. G, Movement kinematics for trials with no, early, and late stimulation. Symbols plot mean values. Error bars indicate SEs, computed across conditions. Initial reach direction was measured relative to target direction. Endpoint variability was measured as the SD of reach endpoint within each condition. 


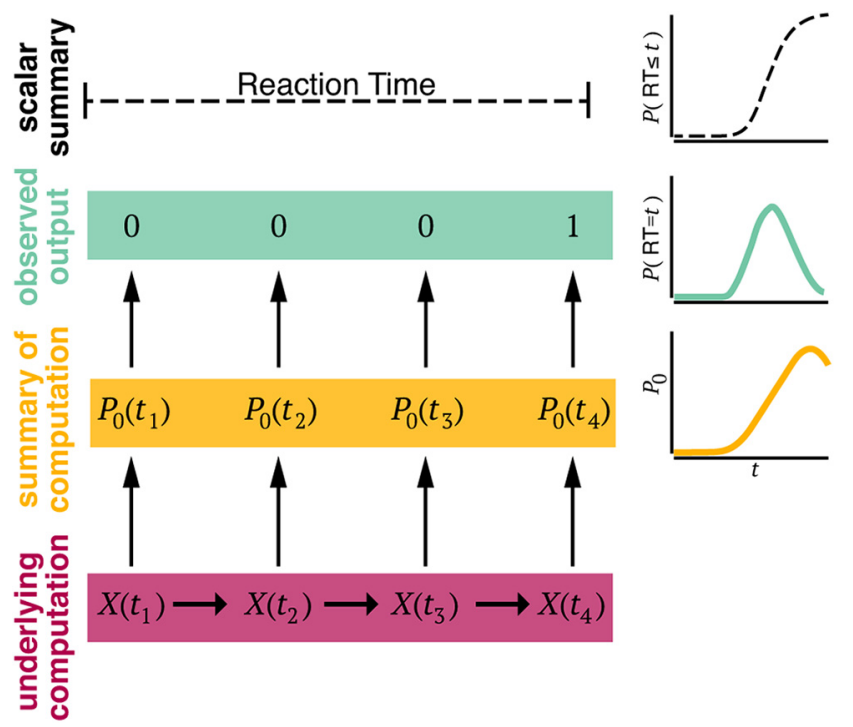

Figure 3. Framework relating the underlying neural state to behavioral measures of movement initiation. The underlying neural state evolves over time (purple box). At each time, there is a probability of initiation, $P_{0}$ (yellow box), produced by that underlying state. (More generally, at a particular time, there is a distribution of possible neural states which determine $P_{0}$ at that time.) The values of $P_{0}$ are not directly observed on a single trial. Rather, one observes multiple time points where initiation does not occur ( 0 in green box) and then a time when initiation does occur ( 1 in green box). These observations are summarized as the RT: the time from when movement was first allowed until it was initiated. Across many trials, one can compute the mean RT, which then becomes a summary of the tendency to initiate. However, a more direct summary can be produced by estimating $P_{0}$ for each time where sufficient data are available.

did not differ between ipsilateral and contralateral targets $(p>$ 0.05 for both monkeys, all contexts, and both early and late stimulation, two-sample $t$ test, calculated across trials).

Thus, SMA microstimulation alters the timing of reach initiation, with negligible impact on the reaches themselves. This is consistent with SMA activity contributing, directly or indirectly, to the computations determining when movement is triggered. SMA stimulation had an effect on all three contexts, arguing that the contribution of SMA to movement initiation is not limited to situations where initiation is less tightly cue-locked. However, the impact of SMA stimulation appears potentially contextdependent. Late-stimulation-driven RT increases were smaller for the quasi-automatic context, and early-stimulation-driven RT decreases were sizeable only for the self-initiated context. Yet as will be detailed below, strong conclusions should not be drawn without first considering that RT may not be the only, or even the most natural, way to measure changes in the tendency to initiate movement.

\section{Movement initiation as a probabilistic process}

The central goal of this study is to characterize how perturbations of SMA activity impact the tendency to initiate and to ask whether that impact is context-dependent. We assume that, at each time, underlying movement-initiating computations have produced a particular neural state (Fig. 3, purple box). That neural state, or more precisely the distribution of such states across trials, yields a probability of initiating at that time. As one example, in a diffusion-to-bound model, initiation becomes probable when the state tends to be close to the bound. We refer to the baseline (without stimulation) probability of initiation at time $t$ as $P_{0}(t)$.

On a given trial, one does not directly observe $P_{0}(t)$. Instead, one observes a stretch of time when movement was not initiated
(" 0 's" in green box) and then a particular time ("1" in green box) when movement was initiated. These observations are often summarized as a single number: the RT. Yet with sufficient trials, one can also estimate $P_{0}(t)$ at each time. To do so, one takes all trials where movement has not yet occurred by time $t$ and computes the proportion where movement is initiated at time $t$. This value is also referred to as the hazard rate.

$P_{0}(t)$ and the RT are intimately related: $P(\mathrm{RT}=t)$ $=P_{0}(t) \prod_{\tau=1: t}\left(1-P_{0}(t-\tau)\right)$. Note that $P(\mathrm{RT}=t)$, unlike $P_{0}(t)$, reflects the entire history of initiation tendencies and thus the entire history of neural states. This creates some interpretational complexities. Suppose that a perturbation alters the neural state at time $t$ and, in doing so, changes the probability of initiation. The resulting change in mean RT is a function not only of that change but of the initiation probabilities at all preceding times. Furthermore, stimulation at time $t_{s}$ implies RT $>t_{s}$, resulting in a longer mean RT even if stimulation has no effect. This can be accounted for (as was done in the analyses in Fig. 2C,D), but doing so adds a layer of complexity. Another complication is that, to properly compare changes in RT, one has to match (across contexts) the time of stimulation. We did so above by defining a common reference time based on when initiation tended to normally occur. This is a reasonable choice, but other reasonable choices are possible. Given these complexities when interpreting changes in the RT, we found it desirable to directly assess changes in the probability of initiation. Doing so allows one to simply inquire, for a given baseline probability, whether one observes a contextdependent change in that probability following stimulation.

\section{Baseline probabilities of initiation}

For each context, we estimated $P_{0}(t)$, the baseline (without stimulation) probability that initiation occurs soon after time $t$, given that it has not occurred before time $t$. We plot that probability as a heat-map (Figs. 4,5, top left, bars). One challenge when assessing $P_{0}(t)$ is a need for high trial counts. For large values of $t$, there may be few trials where movement initiation has not yet occurred, preventing a reasonable estimate of $P_{0}(t)$. Still, by pooling across multiple sites, we were able to estimate $P_{0}(t)$ across a sizeable domain for every context (all non-gray values).

For the self-initiated context, $P_{0}(t)$ remains low for $\sim 600 \mathrm{~ms}$ after target onset (Figs. 4A, 5A; Monkey Ba and Monkey Ax, respectively). The optimal strategy in the self-initiated context is to reach when the presently available reward exceeds the temporally discounted future reward. This idealized strategy would involve selecting a particular time (or equivalently, a particular target size) upon which to initiate, resulting in a step-like increase in $P_{0}(t)$. Monkeys did not follow this strategy; $P_{0}(t)$ increased more gradually, reaching values $>0.9$ only around the time of maximal reward (1200 ms after target onset). Thus, at least from an external perspective, monkeys behaved probabilistically: $P_{0}(t)$ increased monotonically with the juice value of the target.

In the cue-initiated context, $P_{0}(t)$ increased rapidly soon after the go cue (Figs. $4 C, 5 C$ ). For the quasi-automatic context, $P_{0}(t)$ evolved similarly, but the increase was more sudden, occurred slightly earlier, and more swiftly reached values near unity (Figs. $4 E, 5 E)$. This underscores the degree to which initiation was tightly locked to target-motion onset in the quasi-automatic context: $P_{0}(t)$ was essentially unity shortly after motion onset.

The step-like increase in $P_{0}(t)$, especially for the quasiautomatic context, carries potential implications regarding whether perturbations of neural activity have behavioral consequences. As one example, there may be floor/ceiling effects when $P_{0}(t)$ is near zero/unity. Such considerations are a major motiva- 
tion for considering $P_{0}(t)$ directly, rather than relying only on RT measurements. We will return to this point below. For now, we simply stress that the temporal epoch where movement initiation is likely yet uncertain (red zones) has a different duration for the three contexts.

\section{Microstimulation alters initiation probability}

For each context, we estimated $P_{\text {stim }}\left(t, t_{s}\right)$ : the probability that initiation occurs soon after time $t$, given that it has not occurred before time $t$ and given stimulation near time $t_{s}$. We first consider data for Monkey $\mathrm{Ba}$, beginning with the self-initiated context (Fig. 4A). Each row of the bottom heat plot shows $P_{\text {stim }}\left(t, t_{s}\right)$ for a particular value of $t_{s}$. Each such row resembles a slightly altered version of $P_{0}(t)$; differences reflect the impact of stimulation. Early stimulation increased the probability of initiation (the red area extends further leftward). In contrast, late stimulation reduced the probability of initiation (the red area extends further rightward).

To assess these effects, we computed the change in the probability of initiation due to microstimulation: $\Delta P_{\text {stim }}\left(t, t_{s}\right)=$ $P_{\text {stim }}\left(t, t_{s}\right)-P_{0}(t) . \Delta P_{\text {stim }}\left(t, t_{s}\right)$ was not a simple function (Fig. $4 B$ ). There were regions where initiation became more likely (red) and regions where it became less likely (blue). For early stimulation during the selfinitiated context, $\Delta P_{\text {stim }}\left(t, t_{s}\right)$ was largely positive (top rows). Conversely, for late stimulation, $\Delta P_{\text {stim }}\left(t, t_{s}\right)$ was largely negative (bottom rows). This structure agrees with the finding that selfinitiated RTs were advanced by early stimulation and delayed by late stimulation.

For the cue-initiated context, late stimulation extended the region where the probability of initiation was low (Fig. $4 C$, the black trace indicating the 65 th percentile bows to the right). This resulted in negative values of $\Delta P_{\text {stim }}\left(t, t_{s}\right)$ (Fig. $4 D$, blue region). This movement-inhibiting effect agrees with the finding that late stimulation increased RT. Notably, early stimulation had the opposite effect and was movement-promoting (Fig. 4D, red region). This movement-promoting effect is barely detectable at the level of RTs (Fig. 2C) but becomes apparent when assessing $\Delta P_{\text {stim }}\left(t, t_{s}\right)$. Notably, $\Delta P_{\text {stim }}\left(t, t_{s}\right)$ shared some features between the selfinitiated and cue-initiated contexts. In both contexts, early stimulation was movement promoting and late stimulation was movement inhibiting.

For the quasi-automatic context, $\Delta P_{\text {stim }}\left(t, t_{s}\right)$ appears by inspection to differ only slightly from $P_{0}(t)$. In both cases, the probability of initiating underwent a rapid increase, approaching unity, shortly after the go cue (Fig. $4 E$ ). Yet examining $\Delta P_{\text {stim }}\left(t, t_{s}\right)$ reveals that early stimulation was movement promoting (Fig. $4 F$, red regions in top rows) and late stimulation was movement inhibiting (blue regions in bottom rows). These changes are not small, but they are restricted to a narrow temporal window.

For all three contexts, similar effects were observed for Monkey Ax (Fig. 5). One difference was the presence of fewer positive
B

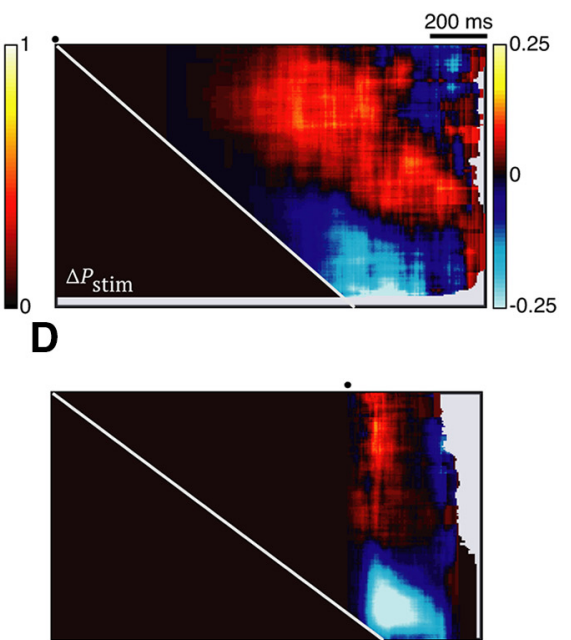

$\mathbf{F}$

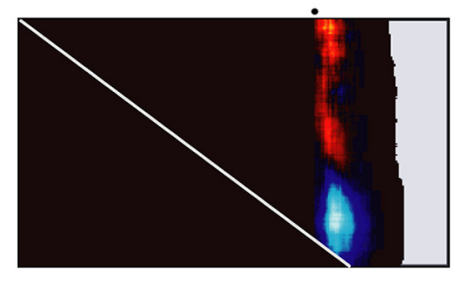

$\stackrel{t}{\longrightarrow}$

Figure 4. Impact of microstimulation on initiation probability for Monkey Ba. $A$, Heat-plots of $P_{0}$ (top) and $P_{\text {stim }}$ (bottom) for the self-initiated context. Time is plotted relative to target onset. For $P_{\text {stim }}(t, t)$, each row plots the probability of initiation, as a function $\Delta P_{\text {stim }}$ for the cue-initiated context. Time is plotted relative to the go cue. $\boldsymbol{E}, \boldsymbol{F}, P_{0}, P_{\text {stim }}$, and $\Delta P_{\text {stim }}$ for the quasi-automatic context. Time is plotted relative to the go cue.

values of $\Delta P_{\text {stim }}\left(t, t_{s}\right)$, especially for the self-initiated and cueinitiated contexts. Thus, movement-promoting effects were less strong in Monkey Ax, consistent with the more modest advancement of self-initiated RTs by early stimulation. Nevertheless, for both monkeys, $\Delta P_{\text {stim }}\left(t, t_{s}\right)$ shared basic features across contexts: early stimulation was largely initiation-promoting and late stimulation was largely initiation-inhibiting.

The above results thus reveal a commonality in $\Delta P_{\text {stim }}$ across contexts. However, all effects were more temporally restricted for the cue-initiated and quasi-automatic contexts, relative to the self-initiated context. A straightforward possibility is that this occurs because only the self-initiated context produces a broad range of times where $P_{0}$ had intermediate values (i.e., neither zero nor unity). Might stimulation have a common impact across contexts, with that impact becoming patent only when the probability of initiation lies within a range amenable to modulation? We explore this possibility below.

\section{A simple model captures changes in initiation probability in the self-initiated context}

We considered a simple model where stimulation impacts the odds of initiation. The odds of initiation is the ratio of the probability of initiating to the probability of not initiating: $O_{0}(t)=\frac{P_{0}(t)}{1-P_{0}(t)}$. We model the impact of microstimulation as follows: 
A
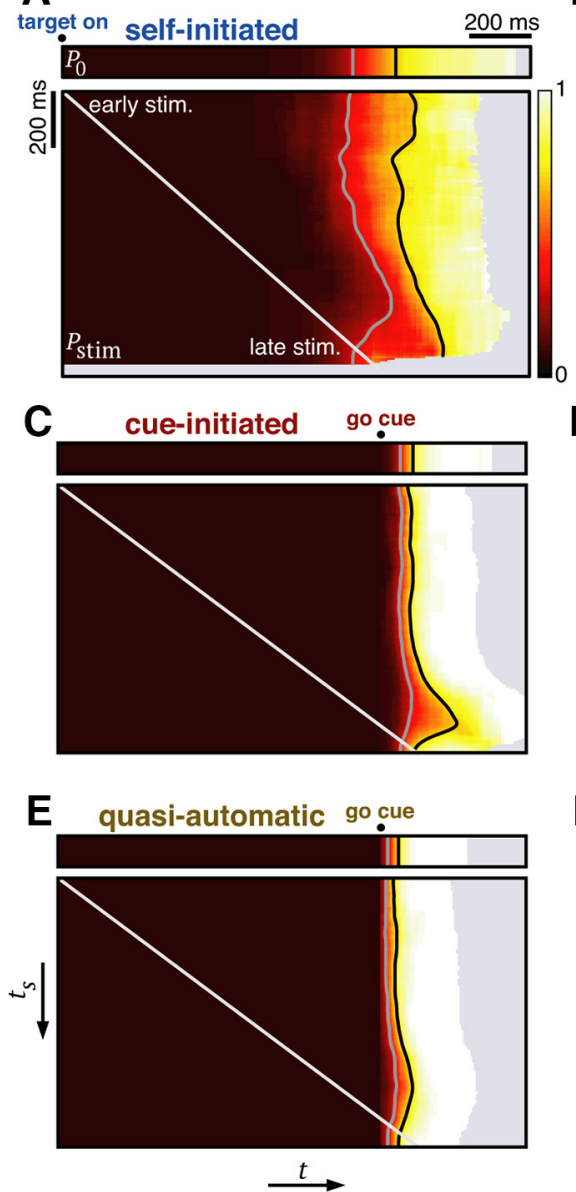

$\mathbf{F}$

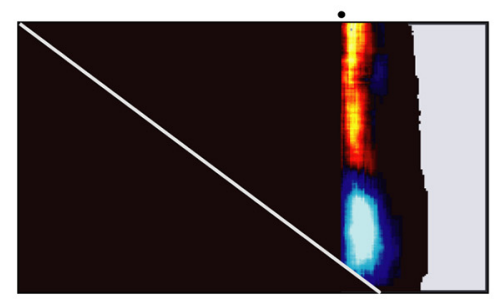

Figure 5. $\boldsymbol{A}-\boldsymbol{F}$, Impact of microstimulation on initiation probability for Monkey Ax. Same format as in Figure 3 .

$$
O_{\text {stim }}\left(t, t_{s}\right)=O_{0}(t) k\left(t-t_{s}\right) .
$$

The kernel $k\left(t-t_{s}\right)$ describes the impact of stimulation, delivered at time $t_{s}$, on the odds of initiating at time $t$. Under this model, the impact of stimulation depends only on the elapsed time since stimulation and not on the overall time within the trial. Using odds provides a natural way of modeling the hypothesis that microstimulation tilts the balance toward or against initiation. The odds of an event can be doubled regardless of baseline probability. For example, for baseline initiation probabilities of $1 \%$, $50 \%$, and $98 \%$ (odds of $1 / 99,1$, and 49 ), doubling the odds would result in poststimulation probabilities of $\sim 2 \%, 67 \%$, and $99 \%$ (odds of 2/99, 2, and 98). The central question is whether it is possible for a single kernel to capture effects across all combinations of $t$ and $t_{s}$. We first considered each context separately, optimized the kernel, and examined the resulting fit.

Figure 6 illustrates model predictions for the self-initiated context for Monkey Ba. Optimization resulted in a kernel with a negative lobe followed by a positive lobe (orange trace at top). To illustrate the impact of this kernel, we first consider the effect of stimulation delivered $220 \mathrm{~ms}$ after target onset (Fig. 6A). Stimulation caused a leftward shift in the empirical probability of initiation: $P_{\text {stim }}$ (black trace) rises earlier than $P_{0}$ (gray trace). This effect is captured by the model (dashed orange trace). The predicted values, $\hat{P}_{\text {stim }}$ exhibit a leftward shift because the positive lobe of $k$ occurs when $P_{0}$ is $>0$ but not yet maximal. Conversely, the negative lobe has little impact because $P_{0}$ is essentially zero at the relevant times. Stimulation $570 \mathrm{~ms}$ after target onset (Fig. 6B) caused a mixed effect: an initial reduction in initiation probability, followed by a small elevation. The model captures this mixed effect, due to the biphasic kernel. Stimulation $740 \mathrm{~ms}$ after target onset (Fig. 6C) caused a rightward shift in $P_{\text {stim. }}$. This is again captured by the model; the negative lobe of $k$ aligns with times when initiation probability is $>0$ but not unity. The fit of $\hat{P}_{\text {stim }}$ to $P_{\text {stim }}$ had an $R^{2}>0.98$ for each of these 3 cases. Thus, effects could be captured by a model where stimulation had an immediate movement-inhibiting effect (decreasing the odds of initiation) followed by a movement-promoting effect (increasing the odds of initiation).

Still focusing on the self-initiated context, we next asked whether fits were similarly good across all tested stimulation times. To provide a more stringent test of the model, we concentrated on the fit to $\Delta P_{\text {stim }}$, rather than $P_{\text {stim }}$ as above. Many features of $P_{\text {stim }}$ are simply inherited from $P_{0}$; the key question is how well one can account for the differences. The model provided a good fit to $\Delta P_{\text {stim }}\left(t, t_{s}\right)$ across the full range of tested values of $t$ and $t_{s}$ (Figs. 7C, $8 C$; compare $\Delta \widehat{P}_{\text {stim }}$ in the middle subpanel with $\Delta P_{\text {stim }}$ in the left subpanel). For both monkeys, $R^{2}$ (Figs. 7, $8 C$, left blue bar) approached an upper benchmark based on the sampling error of the data (gray line, computed via bootstrap; see Materials and Methods). These results confirm that, for the self-initiated context, the impact of SMA microstimulation can be explained by an initial suppression of initiation probability, followed by a weaker facilitation.

\section{Similar kernels explain the impact of stimulation across contexts}

Assessed in terms of RT, SMA stimulation had a contextdependent effect. Is this also true when considering initiation probability? Three results are possible. First, a similar kernel may account for effects across all contexts. Second, the same model may be successful across contexts but may require a different kernel for each. Third, the model may provide a good fit for some contexts but not others, regardless of the kernel used. We found that the first possibility held.

We fit the kernel separately for each monkey and each context. In all cases, the $\Delta \widehat{P}_{\text {stim }}$ produced by the model provided a good fit to the empirical $\Delta P_{\text {stim }}$ (Figs. 7, 8, compare middle and leftmost heat-plots). $R^{2}$ ranged from 0.76 to 0.89 (Figs. 7, 8, bars at right) and was always close to the upper benchmark. The kernels that provided the optimal fit were similar across contexts (Figs. $7 A$, $8 A$ ), even though fitting was independent for each context. In all cases, there was an initial negative phase followed by a shallow positive phase. The correlation of kernel shape between contexts, for a given monkey, ranged from 0.80 to 0.95 . To estimate correlations expected by chance, for each context, we fit kernels to dummy-stimulation data drawn from nonstimulated trials (see 

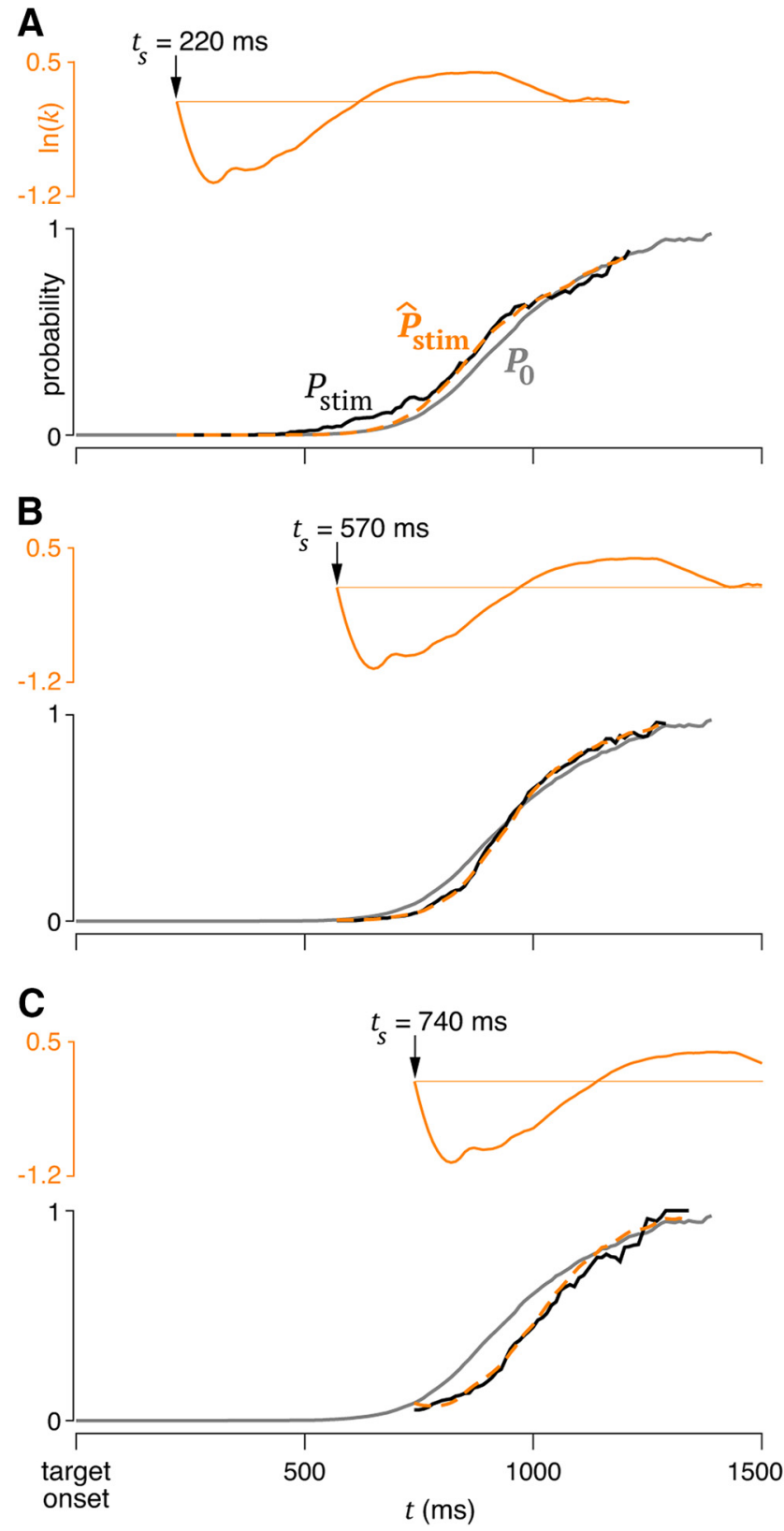

Figure 6. A single, multiplicative kernel predicts $P_{\text {stim }}$ for early, middle, and late stimulation in the self-initiated context. $A$, Data and predictions for stimulation delivered starting $220 \mathrm{~ms}$ after target onset. Gray trace represents $P_{0}(t)$, the baseline probability of initiation without stimulation, for all values of $t$. Black trace represents $P_{\text {stim }}(t, 220)$ : the probability of initiation following stimulation starting $220 \mathrm{~ms}$ after target onset. Dashed orange trace represents $\hat{P}_{\text {stim }}(t, 220)$, the predicted effect of stimulation at that time. This prediction is based on the kernel, $k$, plotted at top (orange trace) aligned to stimulation onset. The log of the kernel is shown, such that a doubling and halving of odds are plotted equidistant from 0 , which indicates no change in odds. $\boldsymbol{B}$, Similar plot for stimulation starting $570 \mathrm{~ms}$ after target onset. The kernel is thus shifted to begin at $570 \mathrm{~ms}$. C, Similar plot for stimulation starting $740 \mathrm{~ms}$ after target onset.

Materials and Methods) and computed the percentage of repetitions where correlations between the three contexts were as high as the empirical values. For both monkeys, empirical correlations were significant $(p<0.001)$.

Consistent with the above results, fit quality decreased only slightly when the model was constrained to use an identical kernel for all contexts (Figs. $7 B, 8 B$, unified kernel). The rightmost heat-plots show the predicted $\Delta \widehat{P}_{\text {stim }}$ using this kernel. Predictions were very similar to those when using a different kernel for each context (middle column). Fits were nearly as good when using the unified kernel as when using context-specific kernels (Figs. 7, 8, compare bars at right). The average reduction in $R^{2}$ was $7.8 \%$. Thus, the same kernel, a negative initial lobe and a subsequent shallow positive lobe, accounted for effects across all contexts. The negative lobe of the kernel was more prominent, but both lobes were important in accounting for effects. We used the resampling procedure described above to ask whether the area within each lobe was greater than expected by chance. This was indeed the case (positive lobe: $p<0.001$ and $p<0.001$; Monkeys Ba and Ax; negative lobe: $p<0.001$ and $p<0.001$ ). Resampling also verifies that effects are due to stimulation itself and not to interactions between behavioral tendencies and incidental properties of stimulated trials (e.g., in the quasi-automatic context, late stimulation implies a longer delay, which could have made monkeys anxious to move).

In summary, effects across all contexts could be accounted for by a simple model where the impact of stimulation depended only on the time since stimulation was delivered. Put differently, we saw no evidence for the hypothesis that perturbation of SMA activity impacts initiation in different ways depending on how tightly initiation is linked to external cues. For a given baseline probability of initiation, the change in that probability due to stimulation was similar regardless of context. This lack of context dependence is potentially surprising. For example, the quasiautomatic context produces highly reactive movements, a situation where initiation might have been expected to bypass any influence of SMA. Put differently, the transition from low to high initiation probability in the quasi-automatic context might have been expected to be unaltered by stimulation. Yet it was altered and in a manner that was captured by the same model that captured effects in the other two contexts. Conversely, the self-initiated context engages internal considerations more heavily, and perturbations of SMA activity might thus have been expected to have a larger impact. Yet this was not the case: changes in initiation probability were consistent with the same model that captured effects in the other contexts.

That said, it certainly is true that a given change in the odds of initiation can impact RT to a greater or lesser degree depending on other factors. For example, the positive lobe of the kernel (which produces an increase in the odds of initiation) will have a larger impact on RT if there is a broad range of times when initiation is possible but far from certain. There is thus no paradox in observing context-independent changes in initiation probability but context-dependent changes in the RT. This is explored quantitatively below.

\section{Kernels that account for initiation probability predict RT changes}

As noted above, the probability of observing a particular RT can be derived from the probability of initiating at each time. This remains true after stimulation: $P\left(\mathrm{RT}=t \mid\right.$ stimulation at $\left.t_{s}\right)$ $=P_{\text {stim }}\left(t, t_{s}\right) \Pi_{\tau=1: t}\left(1-P_{\text {stim }}\left(t-\tau, t_{s}\right)\right)$. We used this relationship to obtain the predicted changes in RT given the values of $\hat{P}_{\text {stim }}\left(t, t_{s}\right)$ produced by the model. To test whether a single kernel could account for the diverse RT effects, we used the unified kernel (Figs. $7 B, 8 B$ ) and computed the predicted RT distribution. We then computed the mean change in RT (relative to no stimulation) for early and late stimulation. The model, although entirely contextindependent, reproduced the context-dependent impact of stimula- 
A

C

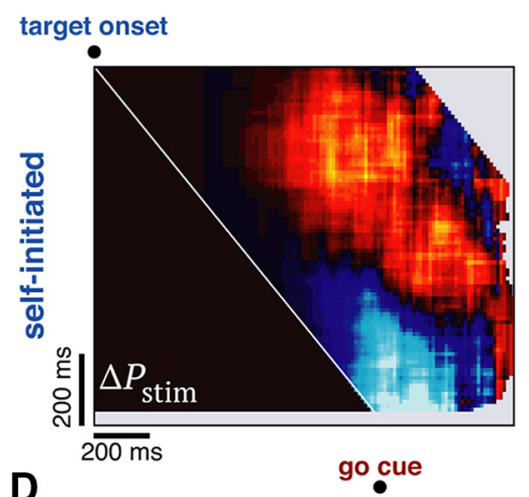

D

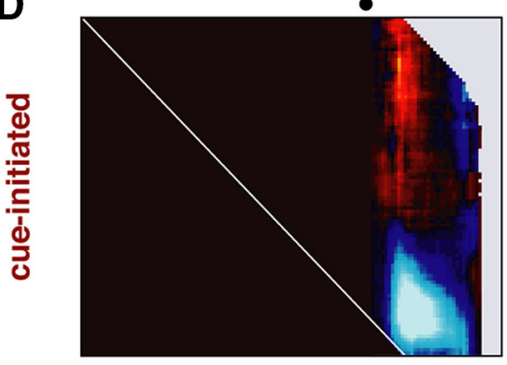

E

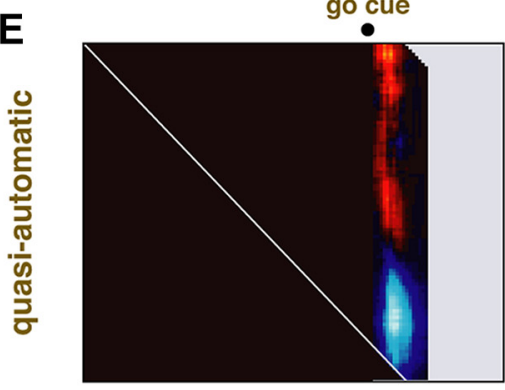

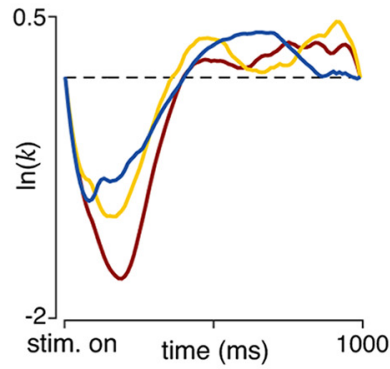

B

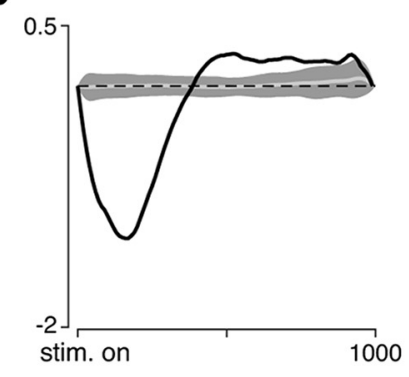

Prediction from context-specific kernel

-
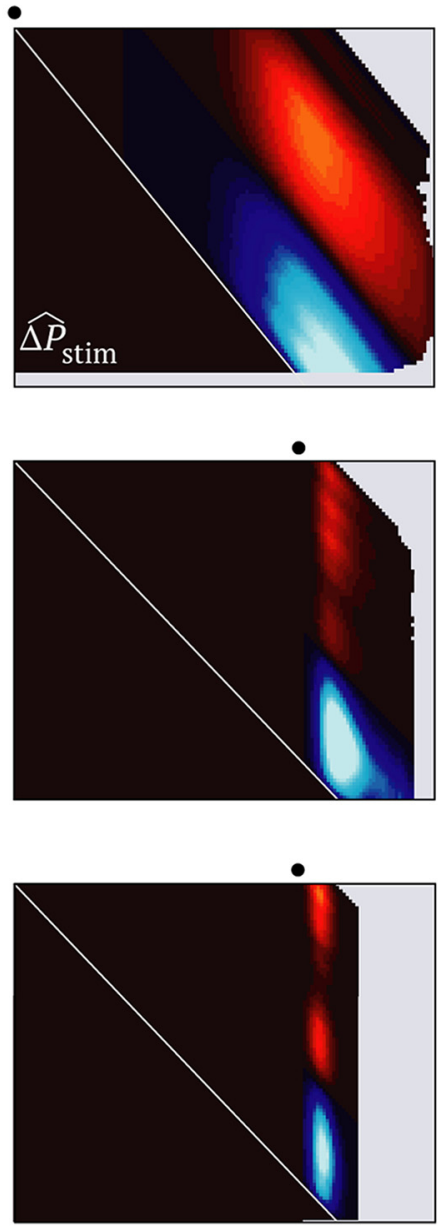

1000
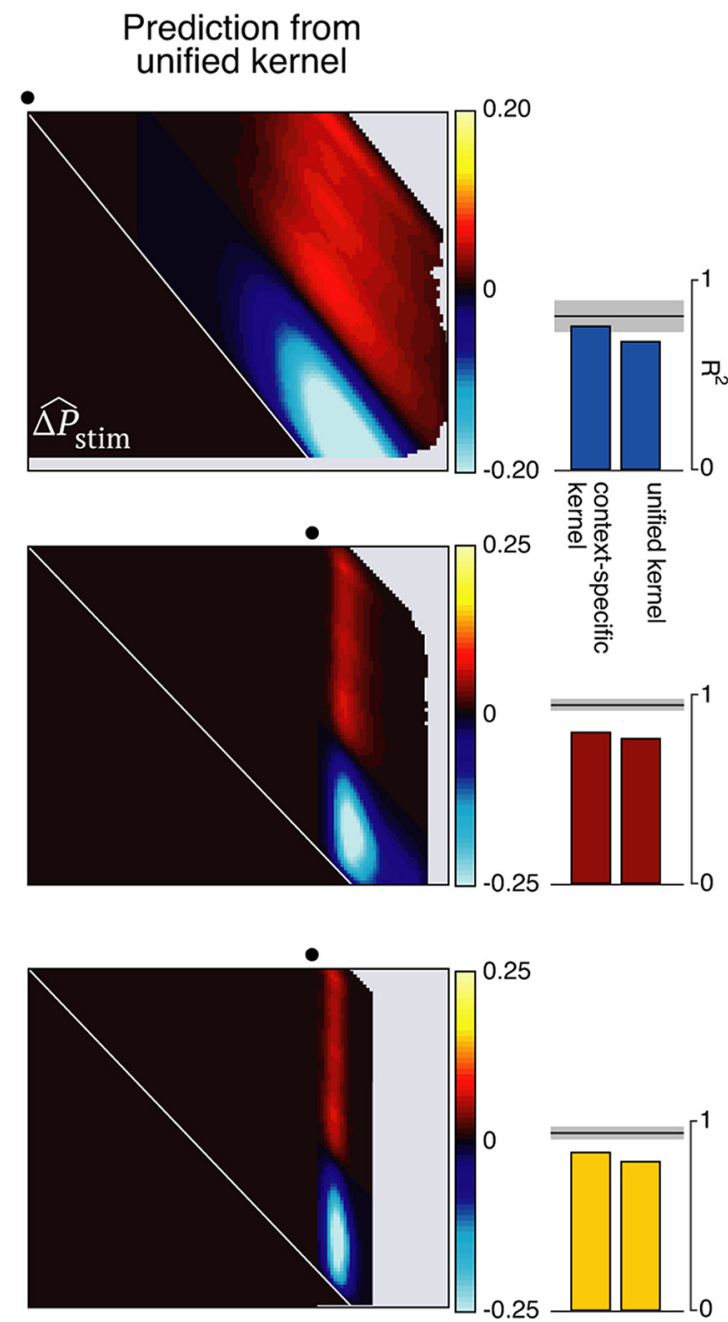

0.25

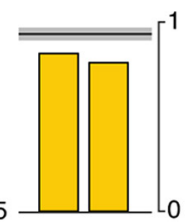

Figure 7. Model predictions of $\Delta P_{\text {stim }}$ for Monkey Ba. $A$, Context-specific kernels, found when fitting to each context separately: self-initiated (blue), cue-initiated (red), and quasi-automatic (yellow). As in Figure 6, the log of the kernel is plotted. Dashed line at 0 thus indicates no change in initiation odds. $\boldsymbol{B}$, Unified kernel, found when fitting to all contexts simultaneously. Shaded region represents $95 \%$ Cls of kernels fit to dummy stimulation data (see Materials and Methods). C, Empirical (left) and predicted changes in the probability of initiation following stimulation in the self-initiated context. Predictions in the middle heat plot are based on the context-specific kernel ( $\boldsymbol{A}$, yellow kernel). Predictions in the right heat plot are based on the unified kernel ( $\boldsymbol{B}$, black kernel). Prediction performance is quantified at right. A rough benchmark on the fit performance that could be provided by a good model was estimated via bootstrap. Horizontal line and shaded region represent mean and $95 \% \mathrm{Cls}$ of that estimate. $\boldsymbol{D}$, Same as for $\boldsymbol{C}$, but for the cue-initiated context. $\boldsymbol{E}$, Same as for $\boldsymbol{C}$, but for the quasi-automatic context.

tion on mean RT (Fig. 9). The variance in $\Delta$ RT accounted for (across the 6 cases for both monkeys) by the fit was 0.88 .

The model captured the advancement of self-initiated RTs following early stimulation. This can be understood in terms of the stimulation-induced change in $P_{\text {stim. }}$. The positive lobe of the stimulation kernel occurs when initiation is gradually becoming likely. A small increase in the odds of initiation during this extended period produces a sizeable change in mean RT. For the other two contexts, the transition from low to high baseline initiation probability is more rapid. Even though these contexts also involve a sizeable increase in initiation probability (e.g., Fig. $8 E$, red regions), the impact on $\mathrm{RT}$ is small due to the restricted temporal window where that effect matters.

The model also captured the increased RT following late stimulation. For late stimulation, the negative lobe of the kernel occurs when initiation is becoming likely. As for the em- 
A

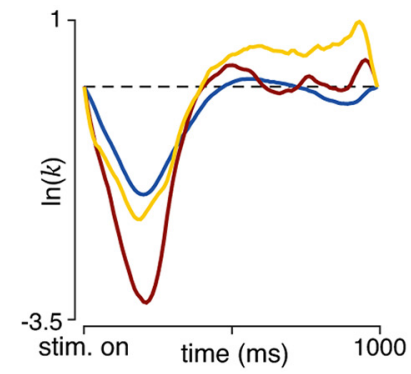

C

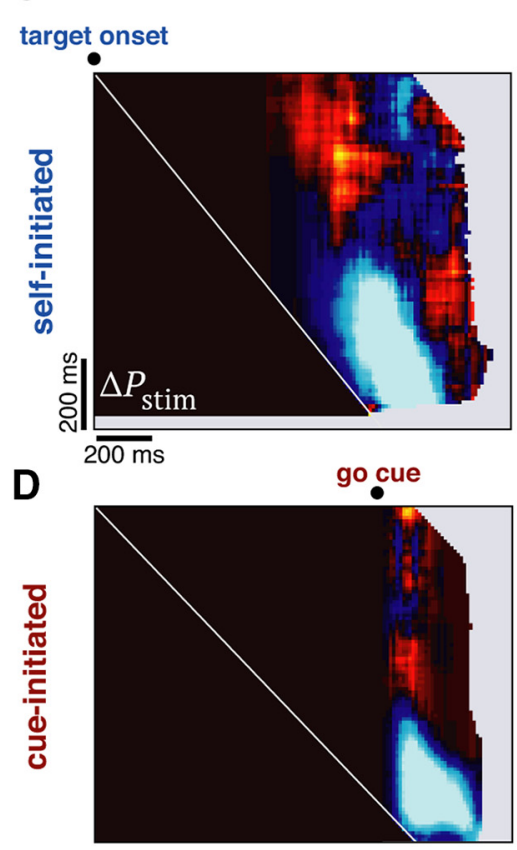

E
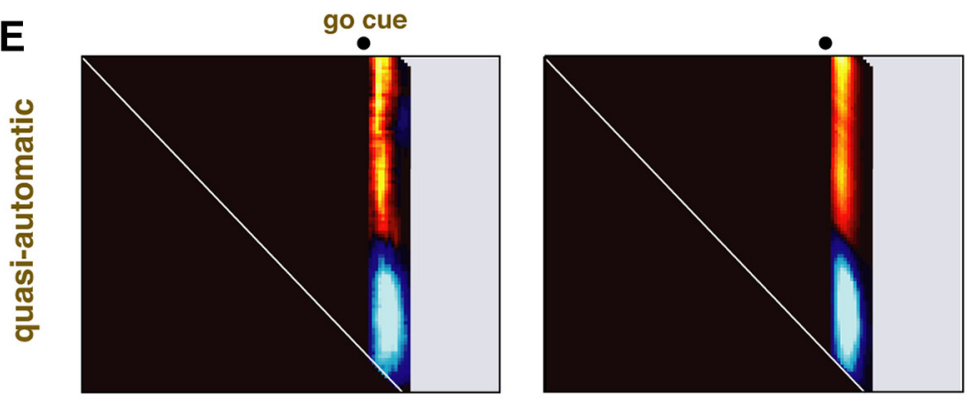

B

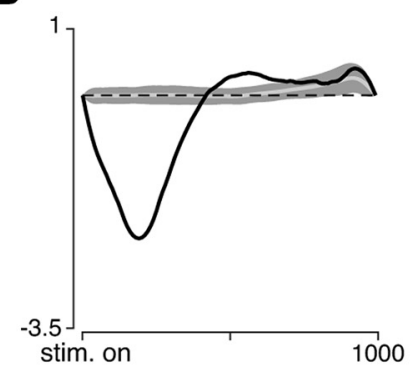

Prediction from context-specific kernel

$\bullet$
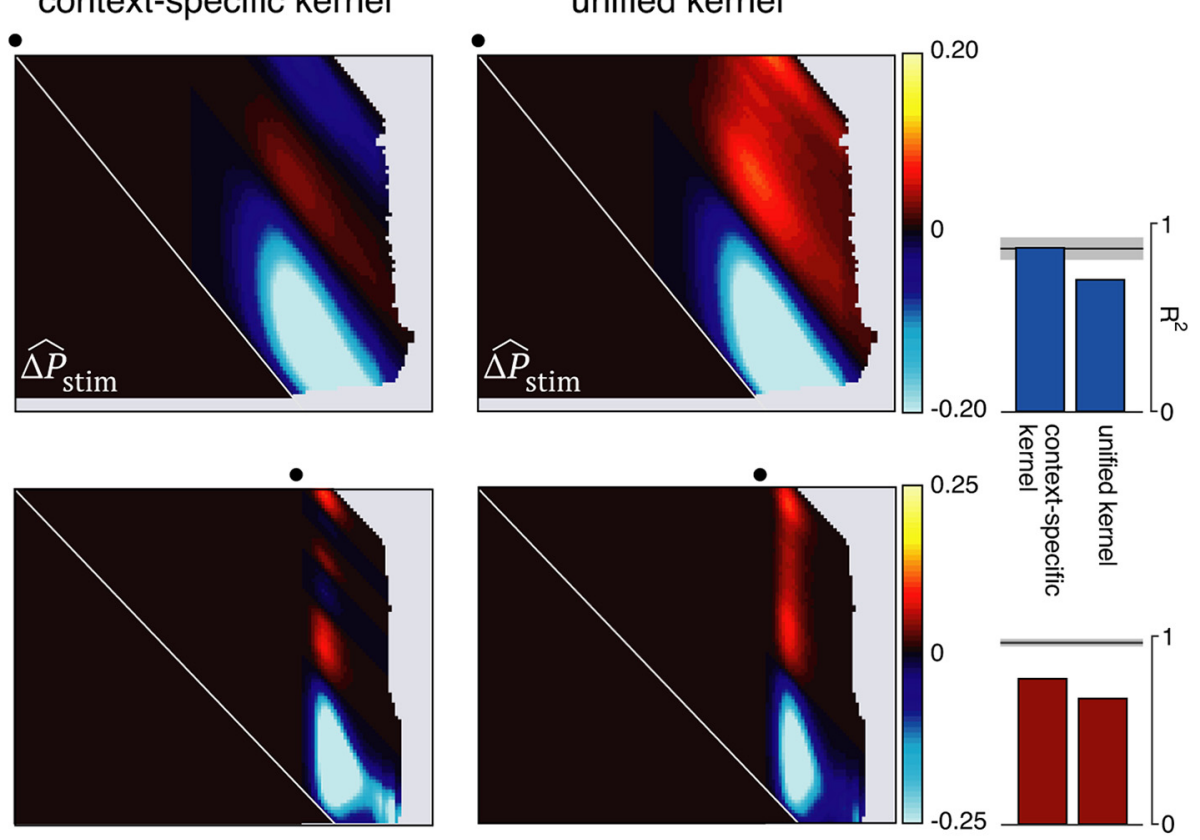

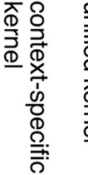

Prediction from unified kernel
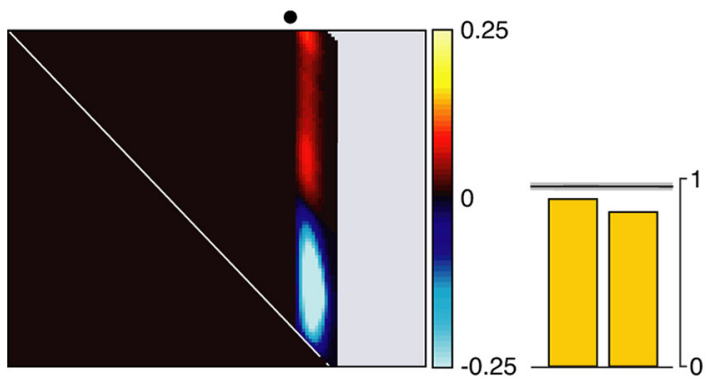

Figure 8. $A-E$, Model predictions of $\Delta P_{\text {stim }}$ for Monkey Ax. Same format as in Figure 7.

pirical data, the RT increase was less pronounced for the quasi-automatic context. This is again a consequence of the rapid increase in baseline initiation probability during the quasiautomatic context.

Despite these successes, the model underestimated some effect sizes. This is unsurprising for two reasons. First, the model reproduces the empirical $\Delta P_{\text {stim }}$ well but not perfectly. Second, the kernel was optimized to minimize mean-squared error between $\Delta P_{\text {stim }}$ and $\Delta \widehat{P}_{\text {stim }}$, rather than $\Delta \mathrm{RT}$ and $\widehat{\Delta \mathrm{RT}}$. If the former error is zero, the latter will also be zero, but in in general the optimal kernel will differ slightly depending on which error is minimized. We can reduce this second contribution by adding, to the cost function, the error between $\Delta \mathrm{RT}$ and $\widehat{\Delta \mathrm{RT}}$, producing a kernel that minimizes both errors. Doing so resulted in small but noticeable improvements in the model predictions of RT $\left(R^{2}\right.$ increased to 0.96 ). For example, the predicted RT reduction in the self-initiated context following early stimulation was 13 and 14 ms larger (Monkeys $\mathrm{Ba}$ and $\mathrm{Ax}$ ) when using the revised cost function, better matching the empirical magnitude.

Thus, a simple context-independent model reproduces the diverse changes in RT. The direction of all effects was correctly predicted, and effect magnitudes were reasonably well predicted. The model assumes that changes in the probability of initiation depend only on the time since stimulation and not on context or the overall time since target onset. Because this parsimonious explanation is naturally expressed in terms of the probability of initiation, subsequent analyses focus on $\Delta P_{\text {stim. }}$. 
A

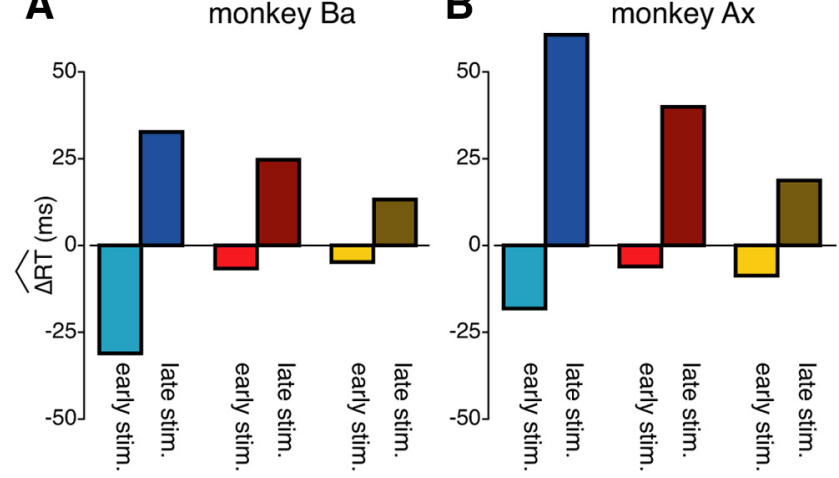

Figure 9. Predicting $\Delta \mathrm{RT}$ from $\hat{P}_{\text {stim. }}$. Predicted $\Delta \mathrm{RT}$ following early or late stimulation. Predictions were made using the unified kernel (found by fitting simultaneously to all contexts). To mirror the analysis in Figure 2, the values of $t_{s}$ used to predict the effect of early and late stimulation were 700 and $300 \mathrm{~ms}$, respectively, before the reference time: the time when movement had begun on $10 \%$ of nonstimulated trials in that context. Because of the $150 \mathrm{~ms}$ window used when originally estimating $\Delta P_{\text {stim }}$, this effectively produced stimulation windows of $700-550 \mathrm{~ms}$ and $300-150 \mathrm{~ms}$ before the reference time, matching the windows used in the original analysis.

\section{Initiation-promoting effects were more prominent for early stimulation trials}

The impact of microstimulation can fade with time (Churchland and Shenoy, 2007). To explore the possibility that some effects may be more prominent when a site is first stimulated, we separately fit stimulation kernels to data for the first 25 stimulation trials at each recorded site (Fig. 10A,E, black kernel). Kernels changed only modestly following this restriction. However, one subtle effect was present for both monkeys: the positive lobe was slightly more prominent when considering only the first 25 trials.

Examination of the data revealed why the fitting procedure used a more prominent positive lobe: positive values of $\Delta P_{\text {stim }}$, corresponding to initiation-promoting effects, were more common when analyzing trials early at a stimulation site. This can be seen by noting that bright red/orange regions are more prominent in Figure 10 (where analysis is restricted to the first 25 stimulation trials) than in Figures 7 and 8 (which consider all trials). This effect was particularly noticeable for Monkey Ax in the selfinitiated and cue-initiated contexts.

The above results predict that the ability to advance RTs, via stimulation early within self-initiated trials, should be enhanced for the first 25 stimulation trials. This was indeed the case. We repeated the analysis in Figure $2 C, D$, restricted to the first 25 trials at each site. RT advancement grew for both monkeys. This increased advancement was sizeable for Monkey Ax (a $24 \pm 9 \mathrm{~ms}$ increase relative to the effect for all trials, $p=$ 0.04 , two-sample $t$ test) and quite modest for Monkey Ba (a $6 \pm 13 \mathrm{~ms}$ increase, $p=0.75$ ). Thus, the movementpromoting impact of SMA stimulation tended to be modestly stronger when a site was first stimulated.

\section{Effects of SMA stimulation across hemispheres and sites}

The kernels above were estimated after pooling data across stimulation sites. Pooling was essential to provide sufficient trials so that $\Delta P_{\text {stim }}\left(t, t_{s}\right)$ could be estimated for every combination of $t$ and $t_{s}$. However, effects may potentially vary across sites. We first considered that sites in one hemisphere might differ systematically from those in the other. This was not the case; kernels were similar when fit separately to data from each hemisphere (Fig. $11 A, B)$. The bilateral impact of stimulation presumably relates to the fact that SMA is active for movements of both the contralateral and ipsilateral arm (Kermadi et al., 1998; Nakayama et al., 2015). However, we note that suprathreshold stimulation typically produced movement only of the contralateral arm. Thus, the initiation-altering effects of stimulation are likely unrelated to the more direct impact of suprathreshold stimulation.

We were also curious whether effects differed across sites within each hemisphere yet found this impossible to address. Trial counts for a single site were insufficient to provide robust estimates of effects, making it unclear whether site-to-site variability was real or due to sampling error. Given this, we stress that the kernels we report should be considered a summary of the central tendency across all sites. Effects at individual sites may (or may not) vary around this central tendency.

Importantly, site-to-site variability did not limit our ability to estimate the central tendency. We resampled our stimulation sites, with replacement, and recomputed the kernel (fitting a single kernel to the data for all contexts). A similar kernel was consistently found, as indicated by the $95 \%$ CIs in Figure $11 C, D$.

\section{Discussion}

SMA has long been thought to play a role in computations determining when and whether to move (Goldberg, 1985; Chen et al., 1995; Thaler et al., 1995; see also Schurger et al., 2012; Murakami et al., 2014; Carlsen et al., 2015; Wang et al., 2018). Our findings validate a key prediction of that hypothesis: manipulation of SMA activity alters the probability of movement initiation. These results likely relate to the prior finding that transcranial stimulation above SMA can, tens of minutes later, impact both RT and the probability of false starts (Carlsen et al., 2015). The timescales involved are very different, yet both sets of results are consistent with perturbation of a computation that influences the likelihood of initiation. A notable feature of the present results is that both increases and decreases in initiation probability were observed depending on stimulation timing. Interestingly, microstimulation of pre-SMA during a saccade task also had a mixed effect: producing shorter RTs when delivered during a delay period yet longer RTs when delivered after the go cue (Isoda, 2005).

It has been suggested that SMA plays a context-dependent role in movement initiation, perhaps being more involved when movements are less linked to external cues and more dependent on internal decisions (Eccles, 1982; Goldberg, 1985; Thaler et al., 1995; Passingham et al., 2010). We observed context-dependent changes in RT, which initially seemed to support that hypothesis. However, changes in the RT can be difficult to interpret for a variety of reasons. As one example, a minimal RT change might indicate that the perturbation had little intrinsic effect, that the effect occurred when initiation was already near certain (a ceiling effect), that the effect occurred when initiation was very unlikely (a floor effect), or that the effect occurred after many trials had already been initiated. The probability of initiation provides a more readily interpretable measure and yielded a more parsimonious summary of effects. Stimulation produced a time-varying change in the odds of movement initiation that was similar across contexts. Indeed, a simple context-independent change in the odds of initiation explained the more complex changes in RT. Thus, we saw no evidence that the impact of SMA stimulation was context-dependent.

We found this lack of context dependence surprising. In particular, quasi-automatic movements were initiated at very low latency, leaving little time for deliberation. For such highly reactive movements, initiation might have been expected to be dissociated from the more cognitive processing typically associated 
with SMA. Yet the same model that accounted for effects in the self-initiated context also accounted for effects in the quasi-automatic context. The contextindependent effect of stimulation was also surprising because SMA activity is strongly context-dependent in this task. Below we consider how this might be possible.

\section{Context-independent stimulation}

effects versus context-dependent activity The finding that SMA stimulation impacts initiation across contexts agrees with the finding that SMA is active across contexts. Thus, both stimulation and recording results (Romo and Schultz, 1987; Kurata and Wise, 1988; Thaler et al., 1988; Picard and Strick, 2003; Hayduk-Costa et al., 2013; Carlsen et al., 2015; Lara et al., $2018 b$ ) argue that SMA contributes to initiation across a broad range of situations. However, the context independence of stimulation effects might appear to conflict with the well-established dependence of SMA neural responses on contextual factors (Tanji and Shima, 1994; Nakamura et al., 1998; Merchant and Georgopoulos, 2006; Merchant et al., 2011, 2013; Nakajima et al., 2013; Merchant and Averbeck, 2017; Lara et al., 2018b; Mendoza et al., 2018; Wang et al., 2018). Indeed, we have confirmed strongly context-dependent responses in SMA (but not motor cortex) in this very same task, at recording sites interspersed among the stimulation sites (Lara et al., 2018b). How could perturbations of contextdependent activity yield context-independent changes in initiation probability?

Although we can only speculate regarding underlying neural events, our results are not paradoxical, as they might first appear. Indeed, the key element, an impact of stimulation that depends primarily on prestimulation initiation probability, emerges naturally given two assumptions. The first assumption is that the impact of stimulation on neural activity is small and not intrinsically context-dependent. This is plausible: stimulation was delivered below threshold, and the same stimulation parameters were used for all contexts. The second assumption is that baseline initiation probability is informative regarding the proportion of trials where the underlying neural state is on the verge of producing initiation.

We assume that there is some "movement-initiating region" of SMA neural state space. This might be the region past threshold in a race model. More generally, it can be thought of as a region where the net influence of SMA becomes strongly movement promoting. At a given time during the task, taking all trials where movement has yet to occur, there will be a distribution of neural states relative to the initiating region. Two hypothetical distributions, corresponding to two contexts, are shown in Figure $12 A, B$. Lighter colors represent baseline distributions. Because distributions correspond to trials where initiation has not yet occurred, they lie largely to the left of the initiating region (gray).

For illustration, distributions have been constructed to be different for the two contexts but to nevertheless have a similar
A

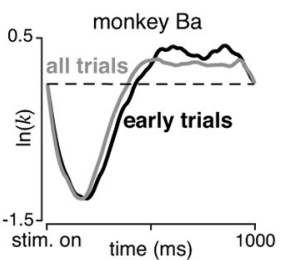

predicted

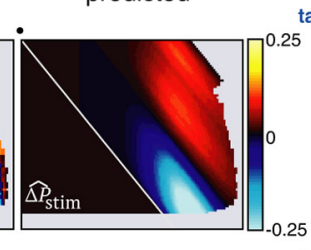

F tar
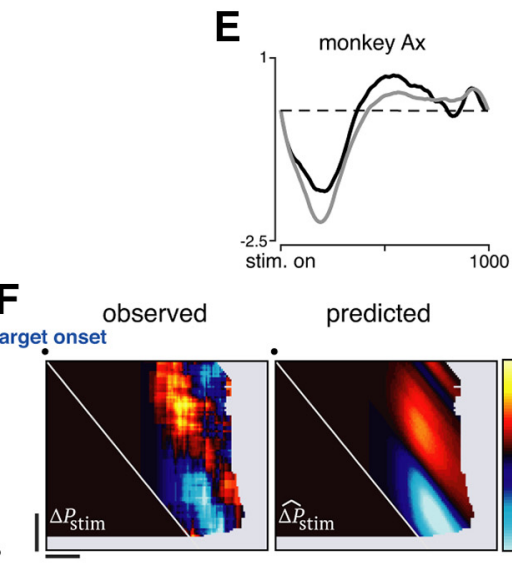

predicted

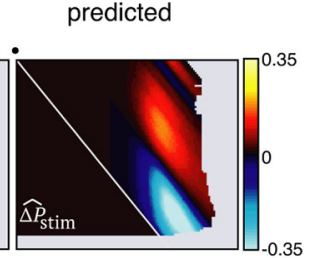

G
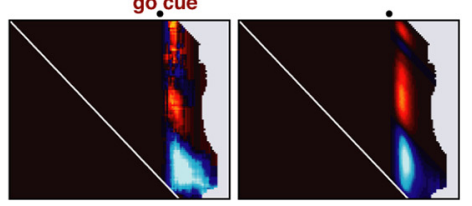

H
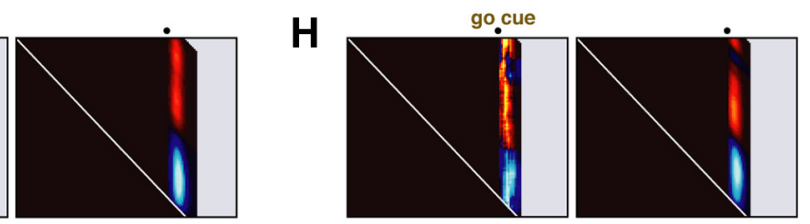

.

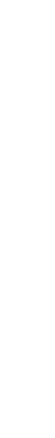
Monkey Ba. $\boldsymbol{E}-\boldsymbol{H}$, Results for Monkey Ax. Kernels were the "unified" kernel fit to all contexts simultaneously. $\boldsymbol{A}$, Stimulation kernel fit to first 25 stimulation trials at each site (black line) and all stimulation trials (gray line). $\boldsymbol{B}$, Empirical (left) and predicted (right) trials at each site. $\boldsymbol{C}$, Same as for $\boldsymbol{B}$, but for the cue-initiated context. $\boldsymbol{D}$, Same as for $\boldsymbol{B}$, but for the quasi-automatic context. $\boldsymbol{E}-\boldsymbol{H}$, Same as for $\boldsymbol{A}-\boldsymbol{D}$, but for Monkey Ax.
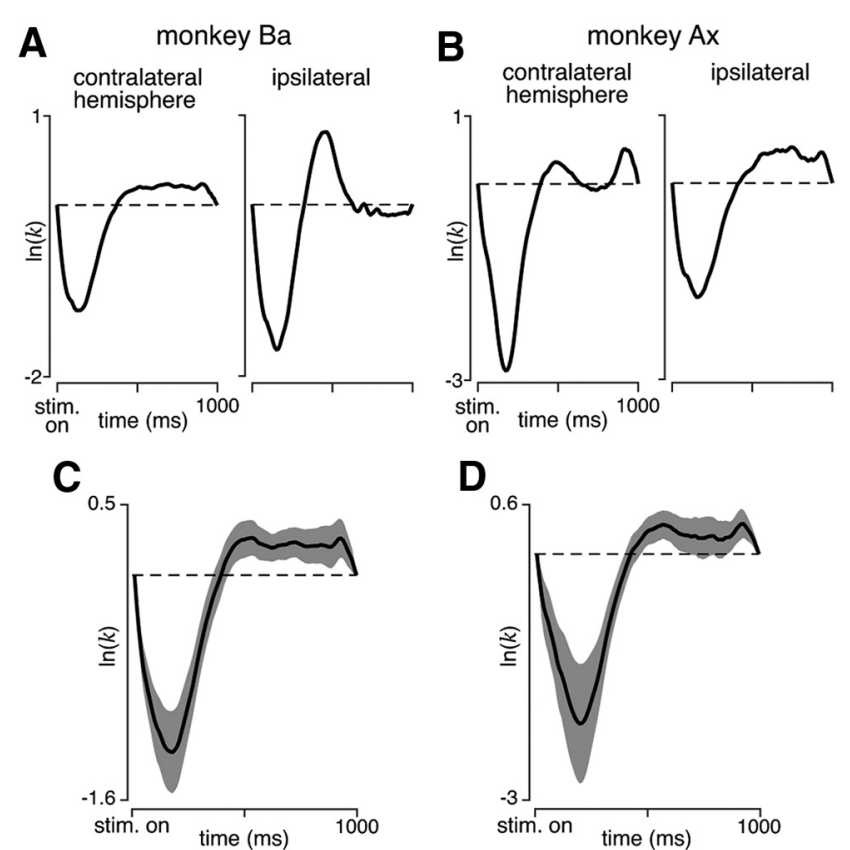

Figure 11. Analysis of potential heterogeneity of effects across sites. $A$, Stimulation kernels for stimulation sites in contralateral (left subpanel) and ipsilateral (right subpanel) SMA. Kernels were the "unified" kernel fit to all contexts simultaneously. Data are for Monkey Ba. $\boldsymbol{B}$, Same as for $\boldsymbol{A}$, but for Monkey Ax. $\boldsymbol{C}$, Reliability of stimulation kernel estimated by resampling stimulation sites. Black trace represents the original stimulation kernel. Gray region represents the $95 \% \mathrm{Cl}$ across resamplings. Data are for Monkey Ba. D, Same as for $\boldsymbol{C}$, but for Monkey Ax. 
A
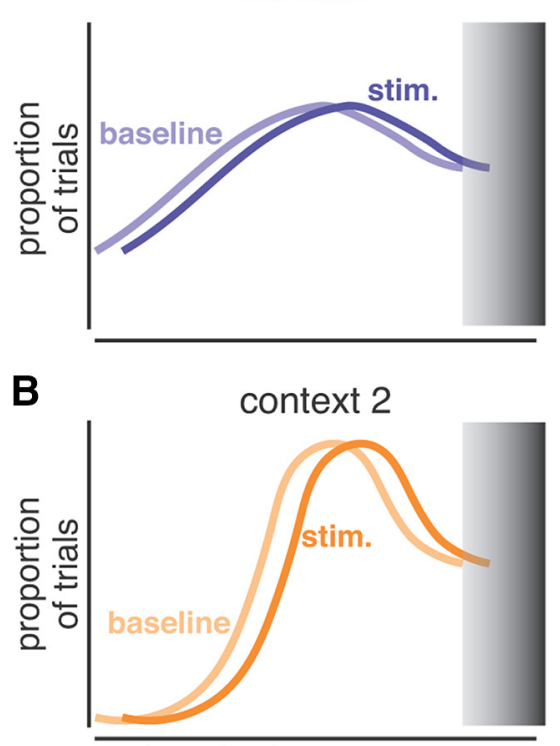

location in state space

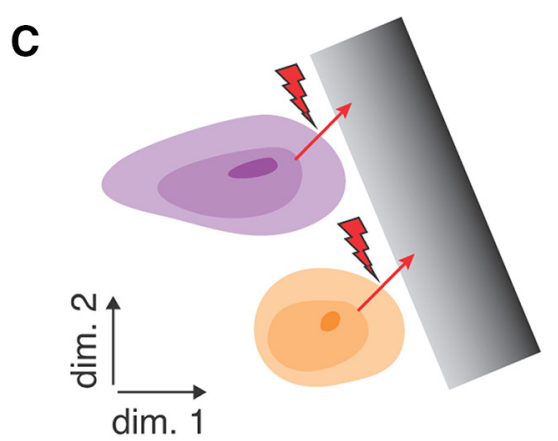

Figure 12. Schematic illustration of how the impact of stimulation may depend primarily on the proportion of neural states near a movement-initiating region. $A$, Possible distribution of neural states in one context. Gray region represents a "movement-initiating region" where activity becomes strongly movement-promoting. Light blue represents a baseline (without stimulation) distribution. A possible movement-promoting impact of stimulation, moving the distribution slightly rightward, is illustrated (dark blue). B, Possible distribution of neural states in another context. Although the baseline distribution differs from than in $A$, there are a similar proportion of states near the movement-initiating region. Thus, the number of trials where the state is "poised" to initiate movement is similar, yielding a similar probability of initiating in the near future. Because the impact of microstimulation is a small rightward shift, the proportion of states shifted into the movement-initiating region is also similar to that in $\boldsymbol{A}$. C, Similar conception but extended to multiple dimensions. Activity is strongly context-dependent. Purple and orange distributions of neural states represent two contexts. Yet the proportion of states shifted into the movement-initiating region is similar in the two contexts.

amount of mass near the movement-initiating region (gray). Thus, both contexts have a similar proportion of trials on the verge of initiation. If we assume that the effect of stimulation is modest (e.g., a small rightward shift as shown), then the proportion of states shifted into the initiating region will be similar in the two contexts. Thus, similar prestimulation initiation probabilities would imply similar poststimulation probabilities, regardless of context. We have simulated a simple race model and confirmed that this is indeed what occurs. Furthermore, it was relatively easy to parameterize that model so that it reproduced our key findings: nearly context-independent changes in initiation probability that produced context-dependent RT changes.

This simple framework is readily extended to multiple dimensions (Fig. 12C). Purple and orange regions represent possible distributions of neural states in two contexts. Thus, activity is strongly context-dependent. However, both distributions have similar mass near the movement-initiating region (gray), consistent with both having similar probabilities of initiation at that moment. If the stimulation-induced change in neural state (red arrow) is small and similar across contexts, the proportion of states driven into the initiating region will be similar. Thus, similar baseline probabilities of initiation will result in similar poststimulation probabilities of initiation, regardless of context.

We stress that context dependence will not hold under all assumptions. Context independence may be lost if the perturbation is large, if the initiating region is curved, or if the impact of the perturbation is not additive with the underlying state. It is thus far from guaranteed that the impact of a perturbation will be context-independent. However, this framework illustrates that there is no intrinsic contradiction between context-dependent activity and context-independent effects of subthreshold stimulation.

\section{Caveats regarding context independence of effects}

Our key analyses combine data across sites for statistical power. Given practical limitations, it was not possible to accurately estimate effect sizes for each context at each site. It thus remains possible that some sites produced greater effects for some contexts, with such variability averaging out across sites. This again underscores that our results should not be taken to rule out the possibility of context-dependent processing in SMA. Rather, our results reveal that perturbation of SMA activity does not, overall, preferentially impact the probability of initiation in some contexts but not others.

Another caveat is that, while our task used contexts where initiation was loosely (self-initiated) or very tightly (quasiautomatic) tied to external cues, this does not span the full range of possible behaviors. In the self-initiated context, monkeys chose when to initiate yet did not have unlimited time. The impact of SMA stimulation on initiation probability could potentially differ in more open-ended situations. At the other extreme, while quasi-automatic reaches were very low-latency, even lowerlatency reaches can be elicited via the startle reflex (Valls-Sole et al., 1995; Carlsen et al., 2004; Honeycutt and Perreault, 2012). It remains unclear whether SMA stimulation would alter the probability of startle-evoked responses, although effects of cathodal transcranial direct current stimulation suggest this is possible (Carlsen et al., 2015). Thus, we cannot conclude that stimulation never interacts with contextual factors. What can be concluded is that the impact of stimulation is surprisingly similar across a range of situations where initiation is either loosely or very tightly linked to external stimuli.

\section{Nature of computation being disrupted}

SMA has been implicated in processes broadly described as executive-control functions, including sequence learning and execution (Tanji and Shima, 1994; Shima and Tanji, 2000; Tanji, 2001), stimulus-response association (Matsuzaka and Tanji, 1996; Hoshi and Tanji, 2004), and conditional response association (Chen et al., 2010). Damage to SMA can produce a lack of spontaneous movement (Laplane et al., 1977; Krainik et al., 2001). Paradoxically, SMA lesions can also cause patients to initiate actions (e.g., don a pair of glasses or pour a glass of water) when contextually inappropriate (the patient was already wearing glasses or was instructed not to pour water) (Feinberg et al., 1992; Boccardi et al., 2002). These findings suggest that SMA plays a role in cognitive processing leading up to movement initiation. 
The present findings support that hypothesis, but the nature of the computation impacted by stimulation remains unclear. There exist multiple overlapping possibilities. Stimulation may alter computations linking sensory cues with appropriate actions (e.g., "a blue target means wait to obtain a large reward") (Nachev et al., 2008). Stimulation may disrupt motor preparation, as occurs in premotor cortex (Churchland and Shenoy, 2007). Stimulation may alter computations related to interval timing (Mita et al., 2009; Merchant et al., 2013; Merchant and de Lafuente, 2014; Cadena-Valencia et al., 2018) or time discrimination (Mendoza et al., 2018). Producing appropriately timed movements, in different ways for the different contexts, is a key aspect of our task.

Alternatively, stimulation could directly impact signals that prompt initiation. This possibility is suggested by the recent finding that SMA and motor cortex share a movement- and contextindependent response component that undergoes a large change just before movement onset (Kaufman et al., 2016; Lara et al., 2018 b), in agreement with theoretical predictions regarding how a neural "trigger signal" might initiate movement generation (Sussillo et al., 2015).

Any or all of the above processes could be altered by stimulation. Perturbation of more than one process might explain the biphasic impact of stimulation (decreasing and then increasing the odds of initiation). Alternatively, the physiological impact of stimulation may be intrinsically biphasic: an initial effect followed by a rebound. In summary, the reasons why SMA stimulation alters the probability of initiation remain unclear. What is clear is that SMA computations influence movement initiation and do so not only in situations where SMA is classically thought to be necessary but across a broad range of contexts.

\section{References}

Boccardi E, Della Sala S, Motto C, Spinnler H (2002) Utilisation behaviour consequent to bilateral SMA softening. Cortex 38:289-308.

Cadena-Valencia J, García-Garibay O, Merchant H, Jazayeri M, de Lafuente V (2018) Entrainment and maintenance of an internal metronome in supplementary motor area. Elife 7:e38983.

Carlsen AN, Chua R, Inglis JT, Sanderson DJ, Franks IM (2004) Can prepared responses be stored subcortically? Exp Brain Res 159:301-309.

Carlsen AN, Eagles JS, MacKinnon CD (2015) Transcranial direct current stimulation over the supplementary motor area modulates the preparatory activation level in the human motor system. Behav Brain Res 279:6875.

Chen X, Scangos KW, Stuphorn V (2010) Supplementary motor area exerts proactive and reactive control of arm movements. J Neurosci 30:1465714675.

Chen YC, Thaler D, Nixon PD, Stern CE, Passingham RE (1995) The functions of the medial premotor cortex: II. The timing and selection of learned movements. Exp Brain Res 102:461-473.

Churchland MM, Shenoy KV (2007) Delay of movement caused by disruption of cortical preparatory activity. J Neurophysiol 97:348-359.

Cunnington R, Windischberger C, Deecke L, Moser E (2002) The preparation and execution of self-initiated and externally-triggered movement: a study of event-related fMRI. Neuroimage 15:373-385.

Eccles JC (1982) The initiation of voluntary movements by the supplementary motor area. Arch Psychiatr Nervenkr 231:423-441.

Feinberg TE, Schindler RJ, Flanagan NG, Haber LD (1992) Two alien hand syndromes. Neurology 42:19-24.

Fried I, Katz A, McCarthy G, Sass KJ, Williamson P, Spencer SS, Spencer DD (1991) Functional organization of human supplementary motor cortex studied by electrical stimulation. J Neurosci 11:3656-3666.

Goldberg G (1985) Supplementary motor area structure and function: review and hypotheses. Behav Brain Sci 8:567-588.

Hayduk-Costa G, Drummond NM, Carlsen AN (2013) Anodal tDCS over SMA decreases the probability of withholding an anticipated action. Behav Brain Res 257:208-214.

Honeycutt CF, Perreault EJ (2012) Planning of ballistic movement following stroke: insights from the startle reflex. PLoS One 7:e43097.
Hoshi E, Tanji J (2004) Differential roles of neuronal activity in the supplementary and presupplementary motor areas: from information retrieval to motor planning and execution. J Neurophysiol 92:3482-3499.

Isoda M (2005) Context-dependent stimulation effects on saccade initiation in the presupplementary motor area of the monkey. J Neurophysiol 93:3016-3022.

Jenkins IH, Jahanshahi M, Jueptner M, Passingham RE, Brooks DJ (2000) Self-initiated versus externally triggered movements: II. The effect of movement predictability on regional cerebral blood flow. Brain 123: $1216-1228$.

Kaufman MT, Seely JS, Sussillo D, Ryu SI, Shenoy KV, Churchland MM (2016) The largest response component in the motor cortex reflects movement timing but not movement type. eNeuro 3:ENEURO.008516.2016.

Kermadi I, Liu Y, Tempini A, Calciati E, Rouiller EM (1998) Neuronal activity in the primate supplementary motor area and the primary motor cortex in relation to spatio-temporal bimanual coordination. Somatosens Mot Res 15:287-308.

Krainik A, Lehéricy S, Duffau H, Vlaicu M, Poupon F, Capelle L, Cornu P, Clemenceau S, Sahel M, Valery CA, Boch AL, Mangin JF, Bihan DL, Marsault C (2001) Role of the supplementary motor area in motor deficit following medial frontal lobe surgery. Neurology 57:871-878.

Kurata K, Wise SP (1988) Premotor and supplementary motor cortex in rhesus monkeys: neuronal activity during externally- and internallyinstructed motor tasks. Exp Brain Res 72:237-248.

Laplane D, Talairach J, Meininger V, Bancaud J, Orgogozo JM (1977) Clinical consequences of corticectomies involving the supplementary motor area in man. J Neurol Sci 34:301-314.

Lara AH, Elsayed GF, Zimnik AJ,Cunningham JP, Churchland MM (2018a) Conservation of preparatory neural events regardless of how movement is initiated. Elife 7:e31826.

Lara AH, Cunningham JP, Churchland MM (2018b) Different population dynamics in the supplementary motor area and motor cortex during reaching. Nat Commun 9:2754.

Macpherson JM, Marangoz C, Miles TS, Wiesendanger M (1982) Microstimulation of the supplementary motor area (SMA) in the awake monkey. Exp Brain Res 45:410-416.

Matsuzaka Y, Tanji J (1996) Changing directions of forthcoming arm movements: neuronal activity in the presupplementary and supplementary motor area of monkey cerebral cortex. J Neurophysiol 76:2327-2342.

Mendoza G, Méndez JC, Pérez O, Prado L, Merchant H (2018) Neural basis for categorical boundaries in the primate pre-SMA during relative categorization of time intervals. Nat Commun 9:1098.

Merchant H, Averbeck BB (2017) The computational and neural basis of rhythmic timing in medial premotor cortex. J Neurosci 37:4552-4564.

Merchant $\mathrm{H}$, de Lafuente $\mathrm{V}$ (2014) Introduction to the neurobiology of interval timing. Adv Exp Med Biol 829:1-13.

Merchant H, Georgopoulos AP (2006) Neurophysiology of perceptual and motor aspects of interception. J Neurophysiol 95:1-13.

Merchant H, Zarco W, Pérez O, Prado L, Bartolo R (2011) Measuring time with different neural chronometers during a synchronization-continuation task. Proc Natl Acad Sci U S A 108:19784-19789.

Merchant H, Pérez O, Zarco W, Gámez J (2013) Interval tuning in the primate medial premotor cortex as a general timing mechanism. J Neurosci 33:9082-9096.

Mita A, Mushiake H, Shima K, Matsuzaka Y, Tanji J (2009) Interval time coding by neurons in the presupplementary and supplementary motor areas. Nat Neurosci 12:502-507.

Mitz AR, Wise SP (1987) The somatotopic organization of the supplementary motor area: intracortical microstimulation mapping. J Neurosci 7:1010-1021.

Moore BD 4th, Aron AR, Tandon N (2018) Closed-loop intracranial stimulation alters movement timing in humans. Brain Stimul 11:886-895.

Murakami M, Vicente MI, Costa GM, Mainen ZF (2014) Neural antecedents of self-initiated actions in secondary motor cortex. Nat Neurosci 17:1574-1582.

Nachev P, Kennard C, Husain M (2008) Functional role of the supplementary and pre-supplementary motor areas. Nat Rev Neurosci 9:856-869.

Nakajima T, Hosaka R, Tsuda I, Tanji J, Mushiake H (2013) Twodimensional representation of action and arm-use sequences in the presupplementary and supplementary motor areas. J Neurosci 33:1553315544. 
Nakamura K, Sakai K, Hikosaka O (1998) Neuronal activity in medial frontal cortex during learning of sequential procedures. J Neurophysiol 80:2671-2687.

Nakayama Y, Yokoyama O, Hoshi E (2015) Distinct neuronal organizations of the caudal cingulate motor area and supplementary motor area in monkeys for ipsilateral and contralateral hand movements. J Neurophysiol 113:2845-2858.

Passingham RE, Bengtsson SL, Lau HC (2010) Medial frontal cortex: from self-generated action to reflection on one's own performance. Trends Cogn Sci 14:16-21.

Perfiliev S, Isa T, Johnels B, Steg G, Wessberg J (2010) Reflexive limb selection and control of reach direction to moving targets in cats, monkeys, and humans. J Neurophysiol 104:2423-2432.

Picard N, Strick PL (2003) Activation of the supplementary motor area (SMA) during performance of visually guided movements. Cereb Cortex 13:977-986.

Romo R, Schultz W (1987) Neuronal activity preceding self-initiated or externally timed arm movements in area 6 of monkey cortex. Exp Brain Res 67:656-662.

Sakata H, Itoh K, Suzuki Y, Nakamura K, Watanabe M, Igarashi H, Nakada T (2017) Slow accumulations of neural activities in multiple cortical regions precede self-initiation of movement: an event-related fMRI study. eNeuro 4:ENEURO.0183-17.2017.

Schurger A, Sitt JD, Dehaene S (2012) An accumulator model for spontaneous neural activity prior to self-initiated movement. Proc Natl Acad Sci U S A 109:E2904-E2913.
Shima K, Tanji J (2000) Neuronal activity in the supplementary and presupplementary motor areas for temporal organization of multiple movements. J Neurophysiol 84:2148-2160.

Sussillo D, Churchland MM, Kaufman MT, Shenoy KV (2015) A neural network that finds a naturalistic solution for the production of muscle activity. Nat Neurosci 18:1025-1033.

Tanji J (2001) Sequential organization of multiple movements: involvement of cortical motor areas. Annu Rev Neurosci 24:631-651.

Tanji J, Shima K (1994) Role for supplementary motor area cells in planning several movements ahead. Nature 371:413-416.

Tanji J, Kurata K, Okano K (1985) The effect of cooling of the supplementary motor cortex and adjacent cortical areas. Exp Brain Res 60:423-426.

Thaler DE, Rolls ET, Passingham RE (1988) Neuronal activity of the supplementary motor area (SMA) during internally and externally triggered wrist movements. Neurosci Lett 93:264-269.

Thaler D, Chen YC, Nixon PD, Stern CE, Passingham RE (1995) The functions of the medial premotor cortex: I. Simple learned movements. Exp Brain Res 102:445-460.

Valls-Solé J, Solé A, Valldeoriola F, Muñoz E, Gonzalez LE, Tolosa ES (1995) Reaction time and acoustic startle in normal human subjects. Neurosci Lett 195:97-100.

Wang J, Narain D, Hosseini EA, Jazayeri M (2018) Flexible timing by temporal scaling of cortical responses. Nat Neurosci 21:102-110.

Wong AL, Goldsmith J, Forrence AD, Haith AM, Krakauer JW (2017) Reaction times can reflect habits rather than computations. Elife 6:e28075. 